\title{
BIRDS
}

\section{9th ANNUAL SASKATCHEWAN CHRISTMAS BIRD COUNT - 2000}

WAYNE C. HARRIS, Saskatchewan Environment and Resource Management, 350 Cheadle Street West, Swift Current, SK S9H 4G3

In spite of rather harsh weather conditions for much of the 2000 Christmas bird count period (December 14 - January 5), 101 counts were completed. This is compared to 99 completed last year during exceptionally mild conditions. It is, however, still less than the 103 from 1997. A total of 804 people participated in this year's count, the most ever. They logged over 650 $\mathrm{km}$ on foot in 360 hours and more than $14,500 \mathrm{~km}$ by vehicle in 608 hours; an additional 610 hours were spent watching feeders (Table 2).

The weather leading up to the 2000 counts was quite varied. First was the heavy snow cover which occurred in the eastern portions of the south in November while the western half remained virtually without snow and remained so through the count period. The second event was the onset of extremely cold weather in early December which only moderated for the last week of the counts.

The overall average temperature was $-17^{\circ} \mathrm{C}$ for a low and $-12^{\circ} \mathrm{C}$ for the high (Table 1). This is deceiving given that most of the counts were completed during the first two weeks of the count period when these averages were close to $-30^{\circ} \mathrm{C}$ for a low and only $-20^{\circ} \mathrm{C}$ for the high. The coldest temperature was recorded at Kamsack where it reached $-40^{\circ} \mathrm{C}$ and the warmest was at Bangor with $8^{\circ} \mathrm{C}$ for a high. The most adverse conditions were from Coronach where the temperature was $-25^{\circ} \mathrm{C}$ combined with a wind up to $70 \mathrm{~km} / \mathrm{hr}$.

The amount of snow was a factor in the eastern half of the province. Both Indian Head and Kennedy reported a metre of snow on the ground. The heavy snow cover was mostly east of a line connecting the Kamsack, Craven and Big Muddy counts.

\section{The birds}

Ninety-five species were recorded this year with all but five seen on count days (Table 3, 4 \&5). In addition there were two recognizable subspecies reported. These numbers are below the average of recent years but are still well above the overall long term average.

Saskatoon, the only count to exceed forty species, reported 42 count day species and three more during the period for an overall total of 45 . Only three other localities reached or exceeded 30 species on their counts (Regina and Fort Walsh, 32; Fort Qu'Appelle 30) which is far below the diversity reported last year when 12 counts had 30 or more species. Overall, the average number of species recorded per count declined by about $20 \%$.

Combined with the decline in the number of species seen on the counts was a decline in the number of individuals. The number of birds seen 
on all counts combined was just over 90,000 , about $40 \%$ less than last year. Snow Buntings were the most abundant species, followed by House Sparrow and Bohemian Waxwing, a more typical ranking especially when compared to last year with Mallard the most abundant species. The most frequently recorded species were Black-billed Magpie and Black-capped Chickadee both, on 95\% of the counts.

On last year's count, redpolls were the highlight species, being reported on more counts and in higher numbers than ever before. In 2000, they were again worthy of specific comment, only exactly opposite to last year. This year redpolls were extremely scarce, being found on less than one third of the counts and the total number of birds was only 749 , both record lows!

\section{Rare species}

For the first time in more than 20 years no new species were reported on this year's counts and the number of rarities was well below normal. Eurasian Collared-Doves, first reported on last year's count, were again present with Regina having one and an additional report from Moose Jaw during count period. These are the third and fourth records for this expanding species. A single male Northern Cardinal was seen at Bangor December 27 (only the sixth record). A Spotted Towhee (formerly Rufous-sided Towhee) at Saskatoon was only the seventh record while the Vesper Sparrow at Whitewood was the third record.

Gray-crowned Rosy Finches were reported from Fort Walsh, the only count where they regularly occur while the one found at Beauval was unusual. Single Brown-headed Cowbirds found at Saskatoon and Turtleford were also unusual as this species is rarely recorded during the winter.

\section{Population trends}

The reduction in numbers of individuals for most species resulted in negative values when populations were compared to past counts for many species (Table 7).

The major event of the counts was the invasion of Great Gray Owls along the southern edge of the boreal forest. Four counts exceeded the previous all time high of 8 birds on a count (Squaw Rapids, 1988). The highest was 18 at Snowden followed by Armit (17), Love (12) and Turtle Lake (11). The counts furthest south recording Great Grays were Whitewood and Broadview. In all, 29 counts reported a total of 111 Great Grays on count day and two other counts had this species during count period. To place this in perspective, in the first 45 years of counts in Saskatchewan only 21 localities recorded Great Grays and the total individuals was 19 !

Other prominent changes were the lower numbers of water related species (Canada Goose, Mallard, Common Goldeneye and Bald Eagle) probably related to the cold weather through much of December and heavy snow cover in the east forcing birds further south than usual.

The other major group showing a decline was the winter finches. Already mentioned was the major decline in Common Redpolls $(-91 \%)$. Hoary Redpolls similarly declined as did both crossbill species. The only species of irruptive finch that increased was Pine Grosbeak.

Forest grouse (Spruce and Ruffed) were down and are now likely at the low in their population cycle. Both Gray Partridge and Ring-necked Pheasant were up in numbers though the winter snow cover in the east is likely to have 
a negative impact during the course of the winter which will show in next year's counts.

The only species to show a significant increase was the Short-eared Owl, likely a result of the lack of snow in the western portions of the province combined with a probable increase in vole and mouse populations. New or tying high counts are given in Table 6 .

Count areas and Participants

(Names of compilers are in italics).

1. ARCHERWILL. Ken Folstad, Judy Revoy.

2. ARMIT. Valeri Harris, Wayne Harris, Sheila Lamont, Bill Zak, Joyce Zak.

3. ASSINIBOIA. Jack Burgeson, Cecil Hayward, Jack Hill, Nelson Lamb, Delmer Pettem, Wilf Prentice, Ken Schuweiler.

4. BANGOR. Allan Bolton, Lynn Bolton, Jean Hilton, Bob Morris, Elinor Morris, Bradley Thompson, Corinna Thompson.

5. BEAUVAL. Clara Sanderson, Rick Sanderson.

6. BETHUNE-DILKE. Doug Laing, Vera Laing.

7. BIGGAR. Dale Booth, Guy Wapple, Marguerite Wapple, Robert Wapple, Sandra Wapple.

8. BIG MUDDY. Martin Bailey, Carol Bjorklund, John Whitell.

9. BIRCH HILLS. Margaret Mareschal, Moe Mareschal, Don Weidl.

10. BRIGHTWATER RESERVOIR. Carman Dodge, D. Peters, Alan Smith, Don Weidl.
11. BROADVIEW. David Chasckavich, Don Weidl, Tony Weidl.

12. BROMHEAD. Martin Bailey, Carol Bjorklund, John Whitell.

\section{CABRI. Carman Dodge.}

14. CANDLE LAKE. Carol Cherkewich, Carman Dodge, Don Weidl.

15. CLARK'S CROSSING. Tony Allen, Lucille Bradatsch, David Cook, Witek Gierulski, Marilyn Haskins, Marlene Kalanack, Richard Kerbes, Gordon Koshinsky, Margaret Koshinsky, Kay Krueger, Gerard Lahey, Menno Nickel, Hilda Noton, Stan Shadick, Wayne Shiels, Marten Stoffel, Karen Wiebe, Michael Williams, Phil Willson, Jim Wood.

16. CORONACH. Wayne Harris.

17. CRAVEN. Betty Binnie, Karen Elliott, Trevor Herriot, Dale Hjertaas, Paule Hjertaas, Phil Holloway, Beth Hone, Bob Kreba, Kevin Moore, Al Sulma.

18. CROOKED LAKE. Vic Greenlaw, Bill Livesay, Mayta Livesay, Boyd Metzler, John Pollock.

19. CYPRESS HILLS PROVINCIAL PARK (Centre Block). Gary Churchill, John Douglas, Shirley Helmerson, Marilyn Scheller, Melody Nagel-Hisey, Tom Pearson.

20. DUCK LAKE. Alan Smith, Don Weidl.

21. DUVAL. Iain Richardson, Lloyd Saul.

22. EASTEND. Robert Gebhardt, Bruce Lewis.

23. EMMA LAKE. Glen Hanson, Jean Hanson, Deanna Krug, Norman Krug. 
24. ENDEAVOUR. Norman Harris.

25. ENDEAVOUR. Ella Smith.

26. ESTEVAN. Marlis Buck, Wayne Harris, Sheila Lamont.

27. ESTON. Bud Code, Barbara Hooper, David Hooper, Ronald Hooper, Darleen Pinter.

28. ESTUARY NORTH. Cathy Cocks, Dean Francis, Frances Hartsook.

29. FENTON. Carman Dodge, Don Weidl.

30. FORT QU'APPELLE. Elizabeth Aitken, James Armstrong, Phyllis Bordass, Anne Davies, Kerrilee Herring, Ronald Hooper, Alice Isfan, John Lamontagne, Lois Lamontagne, Vic Lamontagne, Allan Lewis, John Lowe, Don McDougall, Jean McKenna, Allan Mlazgar, Webb Palmer, Paul Paquin, David Pipes, Lorne Rowell, Elaine Willox.

31. FORT WALSH. Pat Bulman, Wayne Harris, Ron Jensen, Burke Korol, Wilkes Parsonage, Guy Wapple, Rob Wapple.

32. GAINSBOROUGH. Daryl Johannesen, Kaitlin Johannesen, Kristine Johannesen.

33. GARDINER DAM. Pat Bulman, Greg Fenty, Wayne Harris, Stuart Houston, Ron Jensen, Burke Korol, Susan McAdam, Marten Stoffel, Guy Wapple, Rob Wapple, Dan Zazelenchuk.

34. GOOD SPIRIT LAKE. Bill Anaka, Joyce Anaka, Dorothy Riesz, Ray Riesz, Lloyd Wilson, Marge Wilson.

35. GOVENLOCK. Pat Bulman, Wayne Harris, Ron Jensen, Guy Wapple, Rob Wapple.
36. GRASSLANDS NATIONAL PARK. Richard Cherepak, Yolande Cherepak, Bill Fowler, Laura James, Carol Masecar, Cheryl Penny, Colin Schmidt, Brian Spreadbury, Adrian Sturch, Lorie Wiesner.

\section{GRAYSON. John Hicke.}

38. HARRIS. Burke Korol, Guy Wapple, Robert Wapple.

\section{HEPBURN. Phyllis Siemens.}

\section{HOLBIEN. Helen Harris.}

41. HUDSON BAY. Gloria Allchin, Robert Bayet, Tony Binkley, Dan Brann, George Chalus, John Daisley, Mervin Delowski, Ada Hawrish, Agnes Lewellon, Ron Shepard, Antoine Thibedeau, Anae Zaitzsoff.

42. INDIAN HEAD. Chris Blair, Irv Escott, Angela Gehl, David Gehl, Daphine Gehl, Ryan Gehl, Roger Geremia, Gordon Howe, Chris Janrosiacz, Linda Jinks, Dan Loran, Dora Nichols, Pat Nichols, Ann Robb, Lorne Scott, Chris Skinner, Fred Skinner, Ellen Varley, Jack Varley.

43. KAMSACK. John Barisoff, George Bernhard, Hazel Bernhard, Agnes Betz, Marlon Brock, Fred Chernoff, Norma Chernoff, Jeff Conley, Lindee Dewores, Judy Dubasov, Pat Fisher, Bev Helmeyk, Bill Helmeyk, Wanda Kazakoff, Bill Koroluk, Florence Kuzenko, Bernie LaRocque, Laura Lippkey, Adeline Nykolaishen, Bill Poole, Molly Poole, Isabel Ritchie, Metro Rosowsky, Ophelia Sakundiak, Wally Sasyniuk, Bill Severson, Elsie Severson, Bob Smandych, Selma Smandych, Arlin Smorodin, Elenor Sookocheef, Joyce Uhlow, Evelyn Verigin, Peter Verigin, llona Zeiben. 
44. KELVINGTON. Pat Finnie, Dianne Sloan, Marguerite Sloan.

\section{KENASTON. Lawrence Beckie.}

46. KENNEDY. Glen Heebner, Patrick Heebner, Dorothy Hewson, Mae Sawyer.

47. KENOSEE LAKE. Boyd Metzler, John Pollock.

48. KINDERSLEY. Jean Harris, Keith Harris.

49. KINISTINO. Chris Derksen.

50. KINLOCH. Don Forbes, Doreen Forbes, Cliff Logan, Wilf Rodenberg, Doreen Wickstrom.

51. KUTAWAGAN LAKE. Wayne Harris, Allan Rahn.

52. LAIRD. Pearl Bergen, Alice Nickel, Arleyn Nickel.

53. LA RONGE (North). Dianne Allen, Kent Pointon, Whit Pointon, Alice Robert, Keewetin Trottier, Sekwan Trottier, Tekawakin Trottier, Tim Trottier.

54. LA RONGE (South). Dianne Allen, Irene Eriksen, Sharon Feschuk, Ted Green, Hilary Johnstone, Audrey Mark, Joy Picard, Kent Pointon, Sid Robinson, John Schisler, Jan Shewchuk, John Stacey, Marlene Stacey, Tim Trottier, Karen Waters.

55. LAST MOUNTAIN LAKE N.W.A. John Dunlop, Wayne Harris.

56. LEADER (North). Daisy Meyers, George Meyers.

57. LEADER (South). Brenda Flood, John Flood.
58. LOVE - TORCH RIVER. Bert Dalziel, Duke Dalziel, Joan Dalziel, Kari Dalziel, Sara Dalziel, Bruce Donovan, Scott Edwards, Rosanne Kirkpatrick, Eileen L'Heureux, Mildred Long, Bill Matthews, Lynn Matthews.

59. LUSELAND. Steve Allen, Jamie Allen, Estelle Finley, Graeme Finley, Kim Finley, Robert Finley, Val Finley, Bill Frey, Thomas Frick, Brent Honeker, Shirley Honeker, Glen Martfeld, Melba Martfeld.

60. MACDOWALL. Hazel Barton, Myron Barton.

61. MAIDSTONE BRIDGE. Wayne Harris, Sheila Lamont.

62. MEADOW LAKE. Bill Caldwell, Janet Caldwell, Pat Sampson, Joe Twidale, Lorraine Twidale, John Weir, Bob Wilson, Ian Wilson.

63. MELFORT. Aaron Deobald, Calvin Deobald, Irene Deobald, Steven Deobald, Frieda Markland.

64. MISSINIPE. Sid Robinson (nonparticipating compiler), John Schisler, Jan Shewchuk.

65. MOOSE JAW. Elsie Adkins, Jack Adkins, Greg Albert, Pam Albert, Carol Breton, Doug Brunsdon, Helen Brunsdon, Ann Fysh, Pat Kern, Robert Kern, Eve King, Cy Knight, Leith Knight, Arnie Laughlin, Allan Lemieux, Shirley Lemieux, Catherine Letkeman, Doug Manders, Jeff Manders, Bill McDonald, Hugh Mclntyre, Jean Anne Mowchenko, Len Mowchenko, Helen Norys, Peter Norys, Don Parr, Wilma Pickering, Gus Sagall, Mike Sagall, Rose Scheirich, Arnie Van Dorland, Sheina Wait.

66. NAICAM. Eldor Jensen, Julie Jensen, Lauawn Jensen, Ron Jensen. 
67. NIPAWIN. Vi Budd, Joyce Christiansen, Bert Dalziel, Kari Dalziel, Sara Dalziel, Doug Pegg, Helen Pegg, Doug Phillips, Shirley Phillips.

68. NISBET FOREST WEST (NW of Crutwell). Kim Clark, Kiri Clark, Shamara Clark, Suzanne Clark, Bill Marshall, Evelyn Marshall, Jerry Parkin.

69. PADDOCKWOOD. John Dinius, Marg Dinius.

70. PADDOCKWOOD. Blake Jones, Stella Jones, Cliff Matthews, Alice Silversides.

71. PIKE LAKE. Tony Allen, Donna Bruce, Margaret Cato, Ann Christiansen, David Cook, Jill Cornford, Peter Cornford, L. Duczek, Cathy Fry, Greg Fenty, Joe Fry, Bob Girvan, Mike Gollop, Robert Johanson, Marlene Kalanack, Elaine Konkin, Gordon Koshinsky, Margaret Koshinsky, Kay Krueger, Gerard Lahey, Menno Nickel, Michael Olynyk, Keith Pahl, Mary Jean Roy, Marguerite Schneider, Stan Shadick, Angela Stern, Marten Stoffel, Cindy Tkachuck, Richard Tkachuck, Mrs. Torvik, John Ward, Michael Williams, Lois Wooding.

72. PONTEIX. Agnes Kucik, John Kucik.

73. PORCUPINE PLAIN. Agnes Hodge, Keith Larwood, Phyllis Swaan.

74. PREECEVILLE. Frances Buryk, Peter Buryk, Dallas Fairburn, Norman Johnson, Mary Mitchell, Muriel Mitchell, Marie Phoenix, Leona Pollock, Mae Tunbridge.

75. PRINCE ALBERT. John Burt, Pamela Burt, Kim Clark, Carman Dodge, Keith Dodge, Ruth Griffiths, Louise Lenz, Murray Little, Moira McKinnon, Michael Newman, Sheryl Newman, Christine Rye, John Rye, Michael Rye, Peter Rye, Don Weidl.
76. PRINCE ALBERT NATIONAL PARK. Frances Case, Kevin Ferrie, Dan Frandsen, Laura Goreki, Brad Lloyd, Gregg Rutten, Cam Zimmer.

77. QU'APPELLE VALLEY DAM. Dave Cook, Witek Gierulski, Cameron Lockerbie, Stan Shadick, Michael Williams.

78. RAYMORE. Wayne Harris, Sheila Lamont.

79. REGINA. Charles Audison, Jean Audison, Sandy Ayer, Margaret Belcher, Lionel Bonneville, Sarah Bonneville, Stephane Bonneville, Paul Chytyk, Robert Creswell, Jim Cumming, Diane Farmer, Susan Graham, Trevor Herriot, Dale Hjertaas, Paule Hjertaas, Phyllis Ilsley, Bob Kreba, Linda Langenbacher, Rheal Laroche, Catherine Ann Marr, Mervin McElree, Ron Meyers, Caroline Pepper, Wayne Pepper, Giselle Piotrowski, Gunther Piotrowski, Brian Rainey, Karyn Scalise, Diane Secoy, Michael Soroski, Delores Steel, Frank Switzer, Audrey Tranquair, Pat Varls, Michelle Williamson.

80. ROUND LAKE (Qu'Appelle Valley). Doug Francis, Boyd Metzler, Erv Miller, John Pollock.

81. SASKATCHEWAN LANDING PROVINCIAL PARK. Wayne Harris, Ron Jensen, Sig Jordheim, Jim Knelson, Cameron Lockerbie, Sue McAdam, Mike Redford.

\section{SASKATCHEWAN RIVER FORKS. Carman Dodge, Don Weidl.}

83. SASKATOON. Joan Acton, Don Adams, Darlene Aikman, Nancy Allan, Tony Allen, Juhachi Asai, Kengo Asai, Sumiko Asai, Howard Baxter, Neva Bayliss, Don Beazely, Bob Besant, Joyce Besant, Mark Bidwell, Eveline Boudreau, Keith Bowler, Claire Bullaro, 
Pat Bulman, John Catto, Margaret Catto, Mike Chorney, William Christensen, David Cook, Louise Cook, Paul Coutu, Vi Coutu, Ewen Coxworth, Eileen Dalglish, Lorne Duczek, Lillian Dyck, Ann Dzus, Randi Edmonds, Melanie Elliott, Helen Fast, Ray Fast, Catherine Fry, Joe Fry, Albert Gerard, Andre Gerard, Christoph Gerard, Stephan Gerard, Marshall Gilliland, Mary Gilliland, Bob Godwin, Maddie Gollop, Mike Gollop, Jean Gordon, Bob Green, Anne Hanbidge, Bruce Hanbidge, Helen Hanbidge, John Hanbidge, Katie Hanbidge, Kaija Harris, Tim Haughian, Darlene Hay, Jim Hay, Greta HertzumLarsen, Barry Hodge, Mary Houston, Stuart Houston, Andrea Hull, Kathryn Hull, Peter Hull, Richard Kerbes, Gordon Koshinsky, Margaret Koshinsky, Barbara Kozmyk, Kay Krueger, Gerard Lahey, Anna Leighton, Margaret Leighton, Ted Leighton, Cameron Lockerbie, Audrey MacKenzie, Bill MacKenzie, Louise Roy Mark, Kathy Meeres, Cleve Mellor, Cathryn Miller, David Miller, Muriel Miller, Karen Mochoruk, Len Mochoruk, Menno Nickel, Bruce Noton, Hilda Noton, Randy Olson, Keith Pahl, Amanda Plante, Frank Roy, Andy Salisbury, Lorriene Salisbury, Rob Salisbury, Trish Santo, Maureen Scharf, Al Smith, Angela Stern, Marten Stoffel, Margo Taylor, Phil Taylor, Heather Wagg, Jim Wedgwood, Shirley Wedgwood, George West, Michae/ Williams, Jim Wood, Judy Wood, Lois Wooding, Dan Zazelenchuk.

84. SHAMROCK. Joel Chouinard, Lynn Chouinard, Riley Chouinard, Zeke Chouinard, Donna Heinrichs, Gladys Heinrichs, Jaimee Heinrichs, Sharon Heinrichs, Carol Henderson, Hugh Henry, Bob McNeill, Iris McNeill, Ken Priebe, Myrna Priebe, Charnele Samoisette, Elyse Samoisette.

85. SNOWDEN. Bonny Bailey, Elenor Bodnaruk, Bert Dalziel, Stan Dignean,
Irene Hagel, Lily Kuzniar, Jeanette Olson, Jack Pickett, Karen Preistley, Carol Roberts, Ole Rudd, Vera Schemenauer, Vicky Shwetz, Chris Wall, Marlene White.

86. SOMME. Edwin Billeter, Sharon Birch, Wesley Black, Irene Brehmer, Florence Chase, Lorne Chase, Barbara Hooper, Margaret Hooper, Ronald Hooper, Elsie Kerr.

87. SPALDING. Olwen Hoffman, Larry Hoffman, Dora Knutson, Bill Spizawka, Kerry Spizawka, Velma Spizawka.

88. SQUAW RAPIDS. Pat Bulman, Valeri Harris, Wayne Harris, Burke Korol, Sheila Lamont, Guy Wapple.

89. STOCKHOLM. Douglas Flatt, Gordon Smith.

\section{SWIFT CURRENT. Evelyn} Anderson, James Beattie, Jackie Bolton, Merl Campion, Noris Currie, Laurent Dudragne, Mary Ann Dudragne, Michelle Dudragne, Ryan Dudragne, Garland Gould, Joanne Gould, Hugh Henry, Jeff Jensen, Julie Jensen, Ron Jensen, Doreen Kreuger, Walt Kreuger, Arlene Lawson, Doug Lawson, Connie Lendrum, Cameron Lockerbie, Sue McAdam, Eloise Miller, Max Miller, Francis Moen, Ed Nein, Leona Nein, Beth Parchman, Ted Philipchuk, Ernest Schlickting, Maureen Sigmeth, Myra Smith, John Stephenson, Chelsa Stinson, Floyd Stinson, Irene Stinson, Betty Welgan, Ron Welgan, John Weston, Pearl Weston, Carrie Zacharias, Dave Zacharias, Verdella Zacharias.

\section{TISDALE. Carl Mohr, Joyce Mohr.}

92. TOGO. Barb Elsasser, Doug Elsasser, Elsie Elsasser, Roy Elsasser, Claudia Zengl, Conrad Zengl. 
93. TORCH RIVER VALLEY. Laura Blaine, Bruce Donovan, George Lidster, Beatrice Renn.

94. TURTLEFORD. Kristen Kauge, Brent Keen, Wayne King, Jim Mills, David Wooff.

95. TURTLE LAKE. Howard Newton, Ron Poelzer, E.M. Robinson.

96. WALDHEIM. Barb Epp, Winter Epp.

97. WELDON. Ada Hawrish, Bernard Hawrish.

98. WEYBURN. Jean Blackburn, Leo Belanger, Sophie Belanger, Bob Cameron, Ross Douglas, Fred Garner, Lynn Garner, Dick Gutfriend, Betty Layh, Phil Layh, Ken Leblanc, Peggy McDonald, Aaron Schraeder, Doyle Thomas, Val Thomas, Rose Weisgerber.
99. WHITE BEAR. Darryl Jordheim, Sig Jordheim, Elijah MacCulloch, Greg MacCulloch, Lynette MacCulloch.

100. WHITEWOOD. Butch Ashfield, Ellen Blyth, Tom Ede, Wilfred Jordens, Bernice Juzyniec, Felix Juzyniec, Mavis Kay, Doreen McPhail, Jean Meadows, Bill Meszaros, lla Meszaros, Boyd Metzler, Brenda Pollock, John Pollock, Katrina Pollock, Alfred Pritchard, Florence Pritchard, Lenore Santo, Diane Veresh, Ernie Veresh, Pat Ward, Jean Wilson, Ken Wilson, Shag Wilson.

101. YORKTON. Bill Anaka, Joyce Anaka, Dorothy Riesz, Ray Riesz, Geoff Rushowick, Patrick Rushowick, Dorothy Skene.

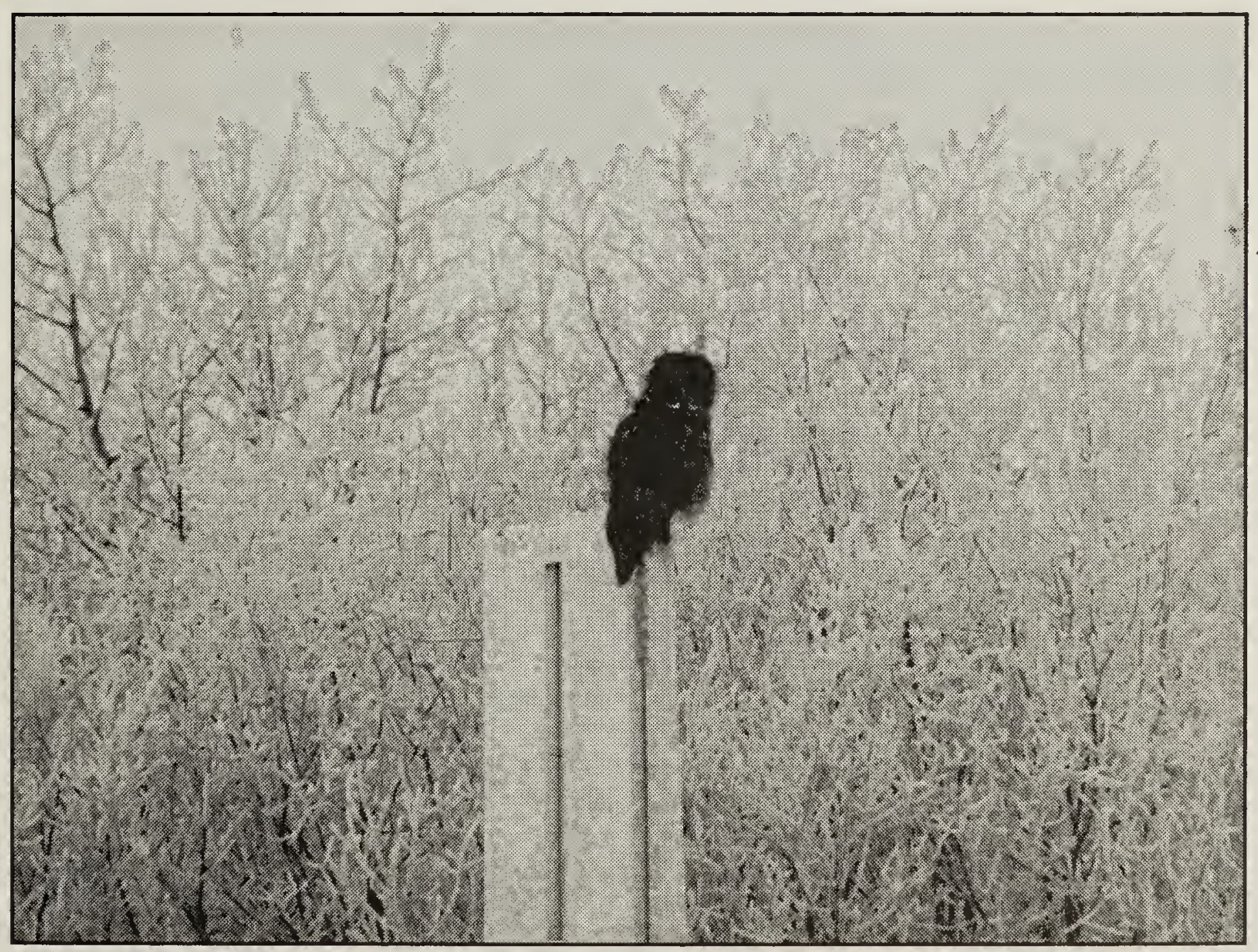


Table 1. Count Weather Conditions

\begin{tabular}{|c|c|c|c|c|c|c|c|c|c|}
\hline Locality & Date & 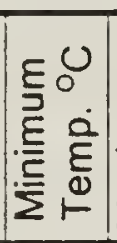 & 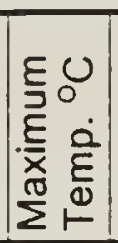 & 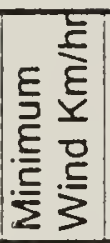 & 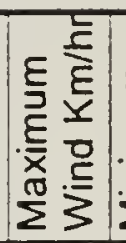 & 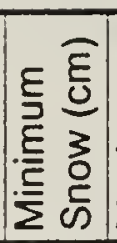 & 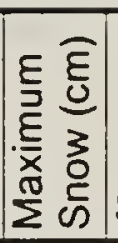 & Sky AM & Sky PM \\
\hline Archerwill & Dec. 27 & -25 & -20 & 5 & 10 & 30 & 35 & clear & clear \\
\hline Armit & Dec. 28 & -15 & -12 & 0 & 10 & 45 & 60 & overcast & overcast, snow \\
\hline Assiniboia & Jan. 4 & -14 & 2 & 10 & 10 & 12 & 15 & mostly clear & mostly clear \\
\hline Bangor & Jan. 3 & 3 & 8 & 5 & 8 & 45 & 50 & mostly clear & partly cloudy \\
\hline Beauval & Jan. 5 & -5 & -3 & 0 & 6 & 20 & 20 & partly cloudy & overcast \\
\hline Bethune-Dilke & Dec. 18 & -21 & -18 & 10 & 20 & 20 & 30 & clear & clear \\
\hline Biggar & Dec. 27 & -15 & -6 & 0 & 40 & 5 & 30 & overcast, rain & overcast \\
\hline Big Muddy & Jan. 3 & -9 & 2 & 0 & 10 & 1 & 20 & mostly clear & mostly clear \\
\hline Birch Hills & Dec. 18 & -28 & -20 & 5 & 15 & 15 & 20 & clear & partly cloudy \\
\hline Bightwater Res. & Jan. 4 & 0 & 2 & 20 & 40 & 15 & 40 & overcast & mostly clear \\
\hline Broadview & Dec. 23 & -32 & -26 & 7 & 12 & 60 & 90 & clear & clear \\
\hline Bromhead & Jan. 1 & -18 & -8 & 20 & 30 & 15 & 25 & clear & clear \\
\hline Cabri & Dec. 23 & -21 & -20 & 0 & 5 & 10 & 15 & mostly clear & mostly clear \\
\hline Candle Lake & Dec. 29 & -16 & -12 & 0 & 5 & 25 & 35 & overcast & overcast, snow \\
\hline Clark's Crossing & Dec. 16 & -33 & -25 & 0 & 10 & 4 & 20 & clear & mostly clear \\
\hline Coronach & Dec. 20 & -25 & -22 & 30 & 70 & 10 & 60 & partly cloudy & mostly clear \\
\hline Craven & Dec. 23 & -25 & -22 & 5 & 10 & 80 & 100 & overcast & overcast \\
\hline Crooked Lake & Dec. 29 & -19 & -14 & 0 & 10 & 40 & 50 & partly cloudy & overcast \\
\hline Cypress Hills & Dec. 29 & -8 & 2 & 0 & 5 & 15 & 40 & mostly clear & partly cloudy \\
\hline Duck Lake & Dec. 15 & -25 & -20 & 10 & 20 & 5 & 15 & overcast & overcast \\
\hline Duval & Dec. 27 & -13 & -8 & 0 & 30 & 15 & 30 & overcast & overcast \\
\hline Eastend & $\operatorname{Jan} .3$ & & 5 & 30 & 50 & 0 & 20 & overcast, fog & overcast, fog \\
\hline Emma Lake & $\operatorname{Jan} .2$ & -4 & -1 & 0 & 5 & 20 & 25 & clear & mostly clear \\
\hline Endeavour & Dec. 25 & -20 & -18 & 0 & 5 & 42 & 50 & overcast & partly cloudy \\
\hline Endeavour & Jan. 1 & -16 & -16 & & & & & clear & clear \\
\hline Estevan & Dec. 26 & -17 & -13 & 0 & 10 & 50 & 60 & partly cloudy & mostly clear \\
\hline Eston & Dec. 26 & -10 & -6 & 8 & 8 & 5 & 8 & light. fog & light. fog \\
\hline Estuary & $\operatorname{Jan} .4$ & & 4 & 0 & 5 & 0 & 15 & partly cloudy & partly cloudy \\
\hline Fenton & Dec. 21 & -32 & -23 & 5 & 15 & 10 & 30 & overcast & overcast \\
\hline Fort Qu'appelle & Jan. 4 & -2 & -2 & 40 & 60 & 40 & 50 & partly cloudy & partly cloudy \\
\hline Fort Walsh & Dec. 16 & -33 & -23 & 10 & 60 & 5 & 30 & partly cloudy & overcast, snow \\
\hline Gainsborough & Dec. 26 & -24 & -22 & 5 & 10 & 30 & 60 & partly cloudy & partly cloudy \\
\hline Gardiner Dam & Dec. 18 & -25 & -20 & 20 & 40 & 0 & 40 & mostly clear & partly cloudy \\
\hline Good Spint Lake & Dec. 31 & -22 & -19 & 2 & 5 & 30 & 42 & mostly clear & mostly clear \\
\hline Govenlock & Dec. 17 & -7 & -5 & 20 & 30 & 0 & 2 & overcast & clear \\
\hline Grasslands N.P. & Dec. 19 & -7 & 0 & 10 & 40 & 1 & 6 & mostly clear & mostly clear \\
\hline Grayson & $\operatorname{Jan} .5$ & -12 & 3 & 14 & 20 & 30 & 35 & partly cloudy & mostly clear \\
\hline Harris & Dec. 21 & -32 & -23 & 10 & 30 & 5 & 20 & clear & clear \\
\hline Hepburn & Dec. 27 & -10 & -8 & 0 & 0 & 15 & 30 & overcast, snow & overcast, snow \\
\hline Holbein & Dec. 29 & -20 & -15 & 0 & 0 & 15 & 24 & overcast & \\
\hline Hudson Bay & Dec. 30 & -20 & -15 & 0 & 0 & 35 & 35 & overcast & \\
\hline Indian Head & Dec. 27 & -10 & -5 & & & 100 & 100 & mostly clear & mostly clear \\
\hline Kamsack & Dec. 26 & -40 & -30 & 0 & 30 & 75 & 75 & clear & mostly clear \\
\hline Kelvington & Dec. 29 & -23 & -19 & 0 & 5 & 25 & 25 & partly cloudy & overcast \\
\hline Kenaston & Dec. 31 & -18 & -9 & 0 & 10 & 20 & 25 & mostly clear & mostly clear \\
\hline Kennedy & Dec. 27 & -16 & -12 & 15 & 20 & 90 & 100 & overcast & overcast, snow \\
\hline Kenosee Lake & Dec. 28 & $\mid-13$ & $\mid-4$ & 20 & 35 & 30 & 70 & overcast & overcast \\
\hline Kindersley & Dec. 31 & -19 & -17 & 0 & 0 & 15 & 20 & clear & clear \\
\hline Kinistino & Jan 5 & -5 & -1 & 1 & 20 & 15 & 100 & overcast & overcast, snow \\
\hline Kinloch & Dec. 29 & -22 & -16 & 0 & 5 & 25 & 301 & partly cloudy & partly cloudy \\
\hline
\end{tabular}


Table 1. Count Weather Conditions

\begin{tabular}{|c|c|c|c|c|c|c|c|c|c|}
\hline Locality & Date & 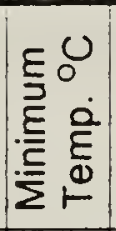 & 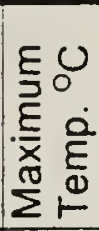 & 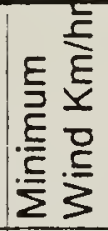 & 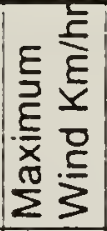 & 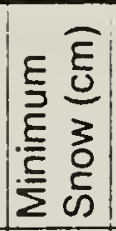 & 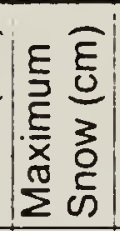 & Sky AM & Sky PM \\
\hline Kutawagan Lake & $\operatorname{Jan} .5$ & 0 & 2 & 0 & 10 & 10 & 30 & overcast & overcast \\
\hline Laird & Jan. 3 & -5 & -5 & 5 & 10 & 12 & 30 & clear & clear \\
\hline La Ronge & Dec. 23 & -35 & -28 & 0 & 10 & 30 & 30 & & clear \\
\hline La ronge (South) & Dec. 31 & -15 & -15 & 0 & 5 & 20 & 30 & mostly clear & mostly clear \\
\hline Last Mt. L. WMA & Dec. 21 & -34 & -20 & 5 & 20 & 0 & 30 & clear & mostly clear \\
\hline Leader (North) & Dec. 30 & -25 & -14 & 2 & 4 & 3 & 20 & partly cloudy & partly cloudy \\
\hline Leader (South) & Dec. 16 & -18 & -13 & 30 & 30 & 8 & 10 & partly cloudy & partly cloudy \\
\hline Love & Dec. 27 & -26 & -20 & 0 & 5 & 25 & 35 & clear & mostly clear \\
\hline Luseland & $\operatorname{Jan} .2$ & -4 & 1 & 0 & 10 & 2 & 25 & partly cloudy & partly cloudy \\
\hline MacDowall & Dec. 28 & -22 & -16 & 3 & 5 & 8 & 15 & partly cloudy & partly cloudy \\
\hline Maidstone & Dec. 31 & -12 & -8 & 0 & 20 & 5 & 20 & mostly clear & mostly clear \\
\hline Meadow Lake & Dec. 26 & -17 & -12 & 15 & 15 & 5 & 10 & partly cloudy & mostly clear \\
\hline Melfort & Dec. 25 & -26 & -20 & 5 & 10 & 5 & 10 & overcast & mostly clear \\
\hline Missinipe & Dec. 31 & -15 & -15 & 0 & 5 & 30 & 30 & mostly clear & mostly clear \\
\hline Moose Jaw & Dec. 26 & -13 & -4 & 2 & 7 & 5 & 20 & partly cloudy & partly cloudy \\
\hline Naicam & Dec. 24 & -23 & -21 & 5 & 46 & 30 & 45 & overcast & mostly clear \\
\hline Nipawin & Dec. 29 & & -24 & 0 & 0 & 22 & 22 & & partly cloudy \\
\hline Nisbet Forest W. & Dec. 26 & -17 & -12 & 0 & 20 & 10 & 20 & overcast & partly cloudy \\
\hline Paddockwood & Dec. 17 & -30 & -28 & 25 & 30 & 30 & 38 & overcast, snow & overcast, snow \\
\hline Paddockwood & $\operatorname{Jan} .4$ & -15 & -11 & 0 & 0 & 27 & 27 & overcast & clear \\
\hline Pike Lake & $\operatorname{Jan} .2$ & -9 & -1 & 0 & 20 & 10 & 40 & partly cloudy & partly cloudy \\
\hline Ponteix & $\operatorname{Jan} .1$ & -7 & -7 & & & & & clear & clear \\
\hline Porcupin Plain & Dec. 29 & -20 & -14 & 0 & 0 & 30 & 35 & overcast & mostly clear \\
\hline Preeceville & Dec. 29 & -20 & -19 & 15 & 20 & 45 & 60 & overcast & overcast \\
\hline Prince Albert & Dec. 17 & -24 & -22 & 0 & 0 & 10 & 20 & overcast & overcast \\
\hline Prince Albert & Dec. 17 & -25 & -20 & 0 & 10 & 10 & 15 & overcast, & overcast, snow \\
\hline Qu'appelle Val. & Dec. 17 & -20 & -15 & 10 & 40 & 7 & 18 & overcast, snow & overcast \\
\hline Raymore & Dec. 25 & -21 & -18 & 10 & 30 & 45 & 60 & overcast & partly cloudy \\
\hline Regina & Dec. 26 & -20 & -11 & 10 & 20 & 30 & 70 & overcast & overcast \\
\hline Round Lake & Dec. 27 & -18 & -10 & 15 & 25 & 24 & 30 & overcast & overcast \\
\hline Sask. Landing & Dec. 19 & -4 & 0 & 10 & 60 & 0 & 10 & partly cloudy & overcast, snow \\
\hline Sask. River & Dec. 19 & -18 & -15 & 10 & 15 & 20 & 30 & overcast & overcast, snow \\
\hline Saskatoon & Dec. 26 & -19 & -14 & 0 & 15 & 10 & 30 & overcast & overcast \\
\hline Shamrock & Dec. 27 & -10 & 4 & 5 & 15 & 8 & 10 & overcast & overcast, rain \\
\hline Snowden & Dec. 30 & -16 & -12 & 0 & 0 & 13 & 30 & overcast, fog & overcast, fog \\
\hline Somme & Dec. 30 & -16 & -12 & 0 & 0 & 30 & 40 & clear & clear \\
\hline Spalding & Dec. 29 & -20 & -15 & 0 & 0 & 30 & 30 & partly cloudy & partly cloudy \\
\hline Squaw Rapids & Dec. 29 & -25 & -18 & 0 & 10 & 15 & 30 & overcast & partly cloudy \\
\hline Stockholm & Dec. 31 & -24 & -20 & 5 & 10 & 15 & 60 & clear & clear \\
\hline Swift Current & Dec. 30 & -14 & -7 & 10 & 25 & 10 & 60 & partly cloudy & partly cloudy \\
\hline Tisdale & Dec. 25 & -10 & -6 & 10 & 15 & 30 & 38 & partly cloudy, & partly cloudy \\
\hline Togo & Dec. 28 & -17 & -14 & 20 & 30 & 50 & 60 & & \\
\hline Torch River & Dec. 22 & -30 & -22 & 0 & 15 & 30 & 40 & & \\
\hline Turtleford & $\operatorname{Jan} .6$ & 2 & 4 & 20 & 20 & 10 & 12 & overcast, snow & overcast, snow \\
\hline Turtle Lake & Dec. 26 & -20 & -10 & 5 & 10. & 10 & 15 & clear & partly cloudy \\
\hline Waldheim & $\operatorname{Jan} .2$ & -5 & 0 & 0 & 0 & 30 & 60 & & mostly clear \\
\hline Weldon & Dec. 17 & -30 & -22 & 18 & 20 & 10 & 15 & overcast, snow & overcast, snow \\
\hline Weyburn & Jan. 3 & -13 & -4 & 0 & 0 & 15 & 60 & mostly clear & partly cloudy \\
\hline White Bear & Dec. 27 & -7 & -2 & 2 & 15 & 5 & 25 & overcast & partly cloudy \\
\hline Whitewood & Dec. 31 & -19 & -14 & 0 & 10 & 20 & 36 & mostly clear & clear \\
\hline Yorkton & Dec. 30 & -19 & -12 & 0 & 0 & & & lovercast, fog & \\
\hline
\end{tabular}


Table 2. Count Coverage

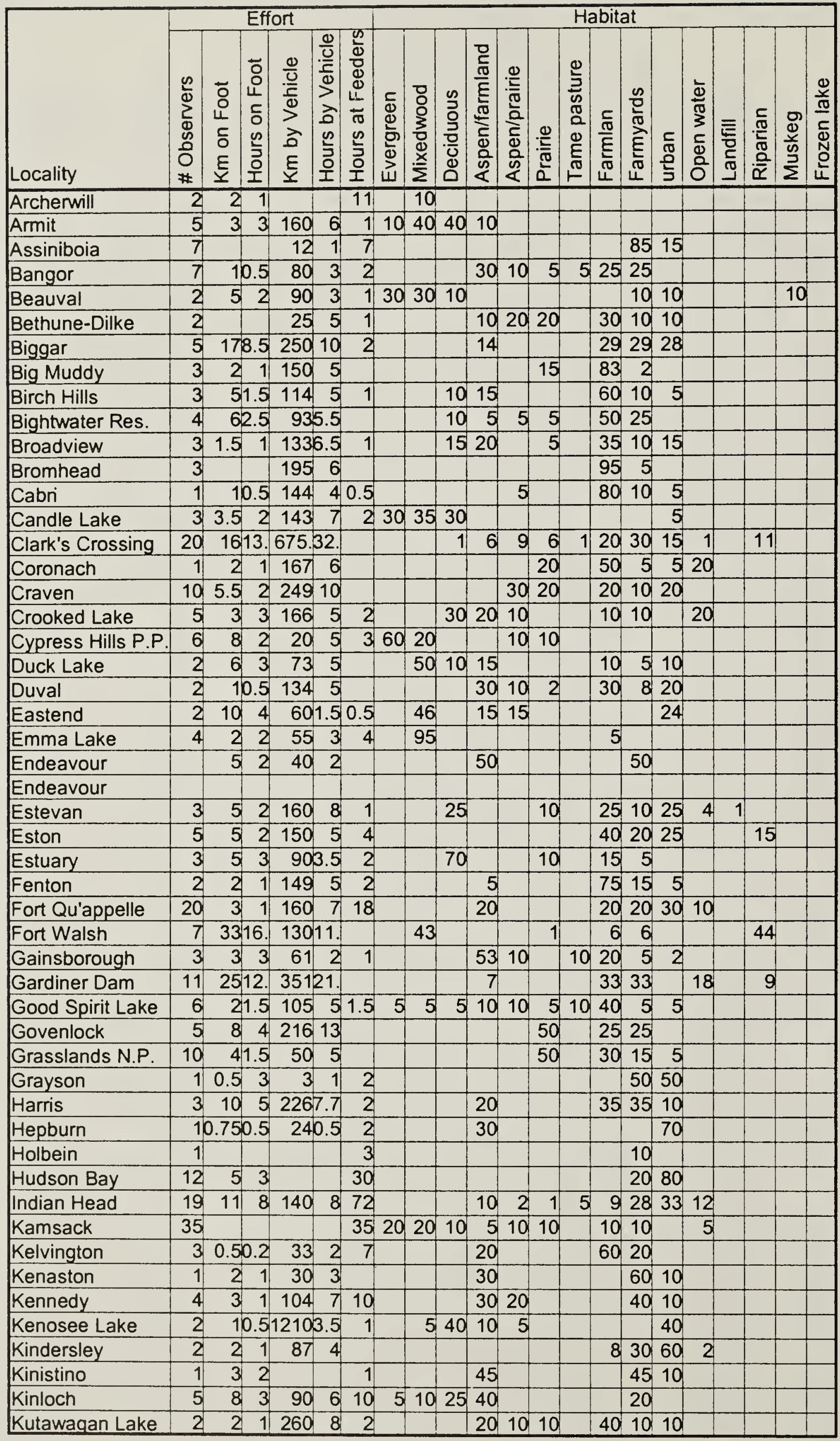


Table 2. Count Coverage

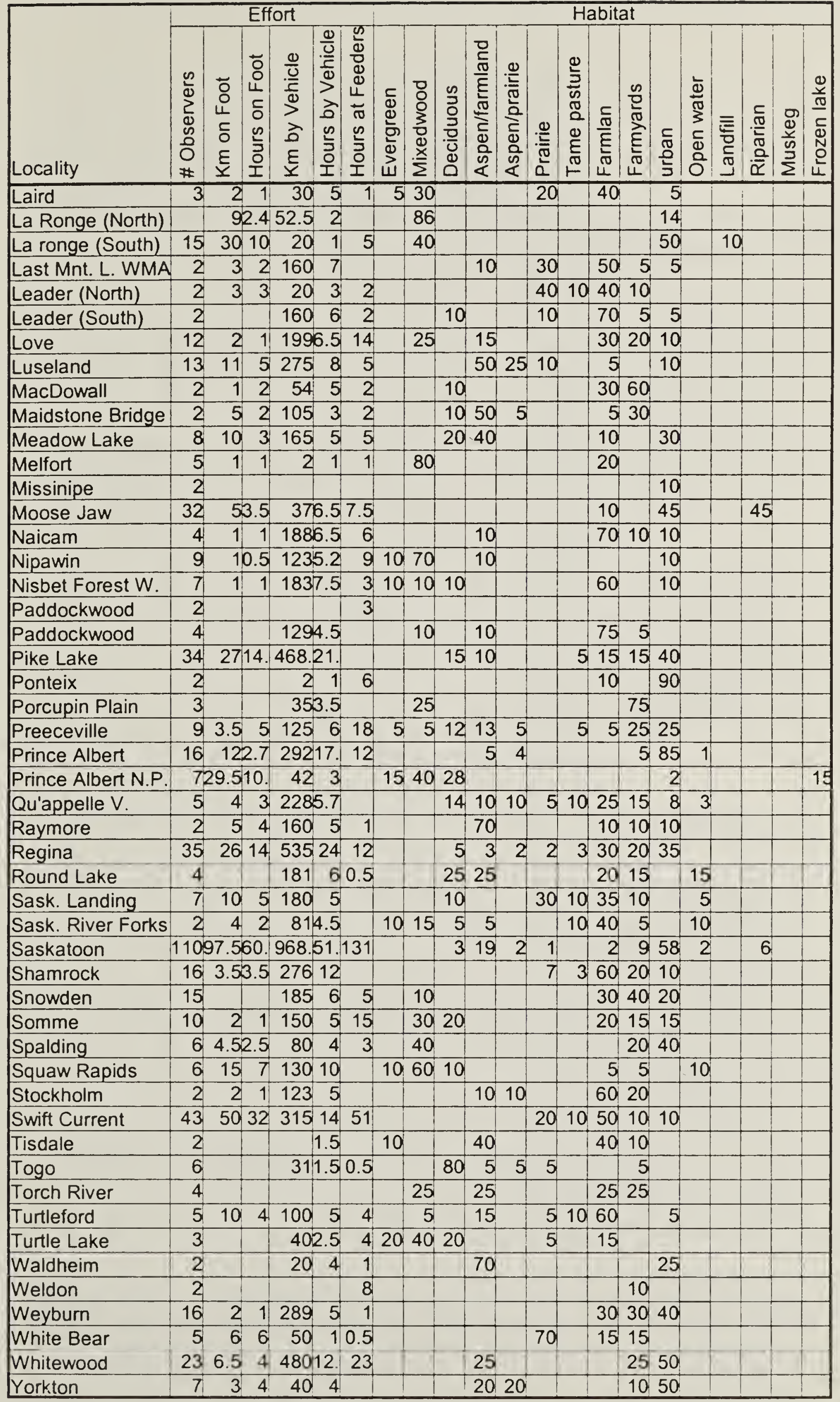


Table 3-1. SPECIES RECORDED FROM NINE OR MORE LOCALITIES

$(0=$ a species recorded during count period but not seen on count day)

SPECIES

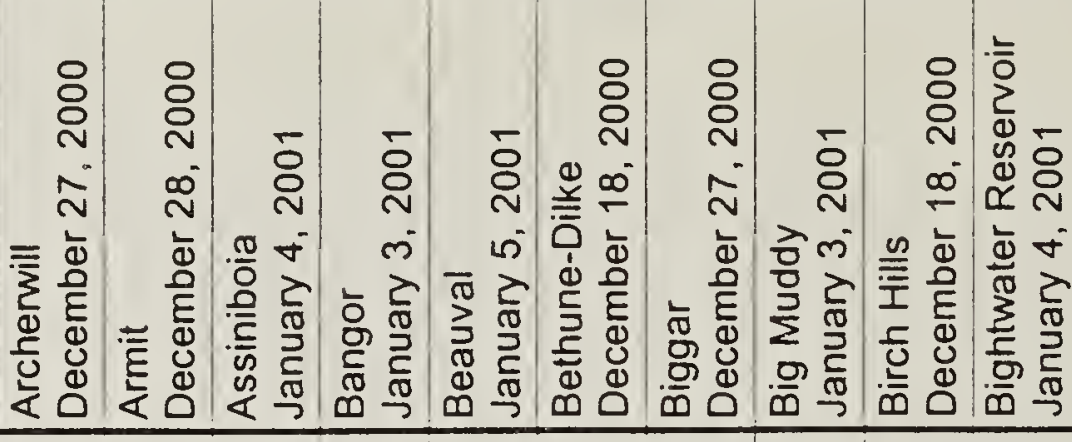

MALLARD

COMMON GOLDENEYE

BALD EAGLE

NORTHERN GOSHAWK

GOLDEN EAGLE

GRAY PARTRIDGE

RING-NECKED PHEASANT

SPRUCE GROUSE

RUFFED GROUSE

SHARP-TAILED GROUSE

ROCK DOVE

GREAT HORNED OWL

SNOWY OWL

GREAT GRAY OWL

SHORT-EARED OWL

DOWNY WOODPECKER

HAIRY WOODPECKER

THREE-TOED WOODPECKER

BLACK-BACKED WOODPECKER

NORTHERN FLICKER (Y-s)

PILEATED WOODPECKER

HORNED LARK

GRAY JAY

BLUE JAY

BLACK-BILLED MAGPIE

AMERICAN CROW

COMMON RAVEN

BLACK-CAPPED CHICKADEE

BOREAL CHICKADEE

RED-BREASTED NUTHATCH

WHITE-BREASTED NUTHATCH

AMERICAN ROBIN

BOHEMIAN WAXWING

CEDAR WAXWING

NORTHERN SHRIKE

EUROPEAN STARLING

HARRIS' SPARROW

DARK-EYED JUNCO (S-C)

SNOW BUNTING

PINE GROSBEAK

HOUSE FINCH

COMMON REDPOLL

PINE SISKIN

EVENING GROSBEAK

HOUSE SPARROW

TOTAL INDIVIDUALS

SPECIES ON COUNT DAY

SPECIES COUNT PERIOD

INDIVIDUALS IN TABLES $4 \& 5$

SPECIES IN TABLES $4 \& 5$

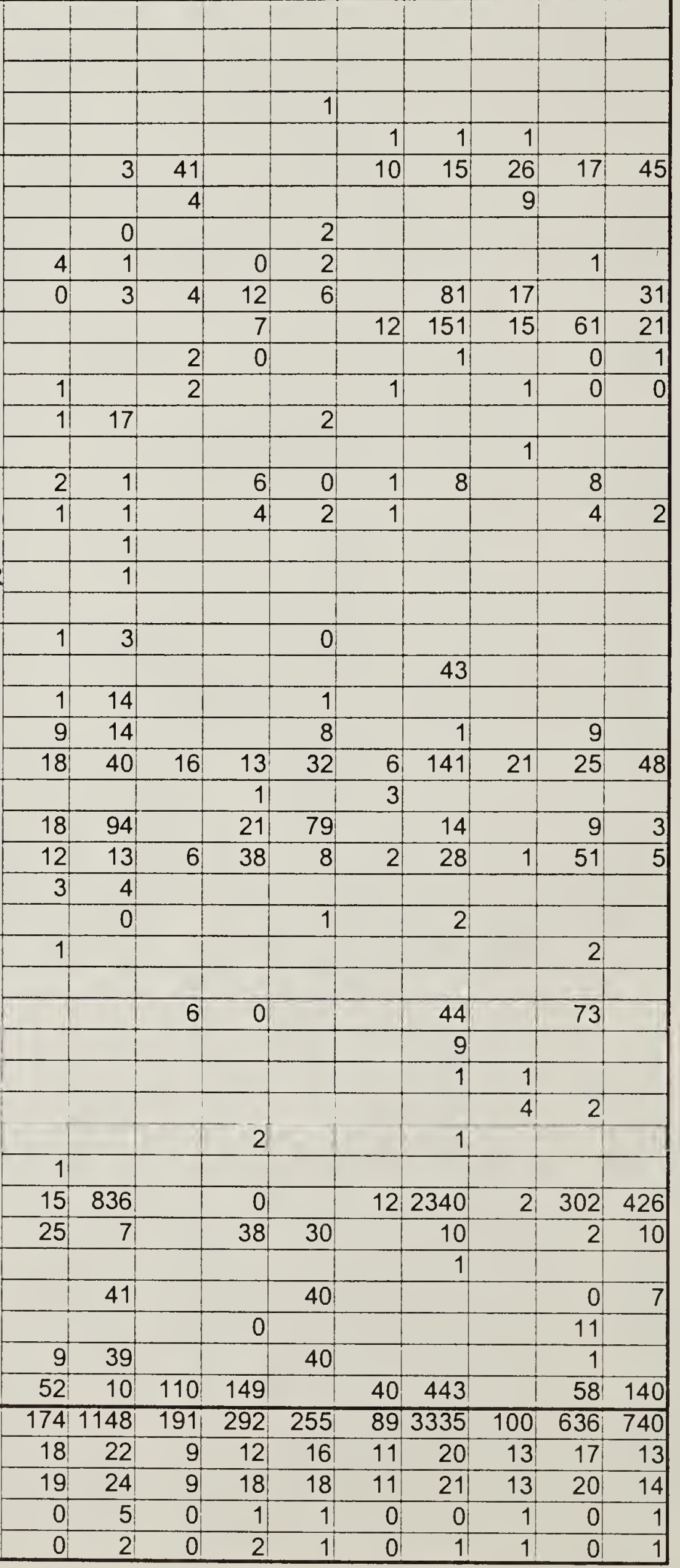


Table 3-2. SPECIES RECORDED FROM NINE OR MORE LOCALITIES $(0=a$ species recorded during count period but not seen on count day)

\begin{tabular}{|c|c|c|c|c|c|c|c|c|c|c|}
\hline 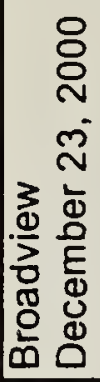 & 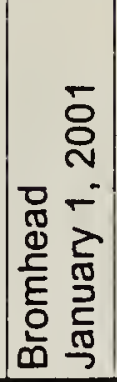 & 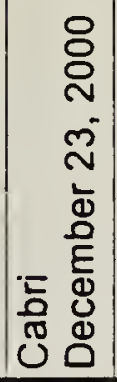 & 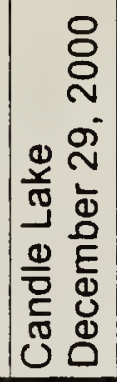 & 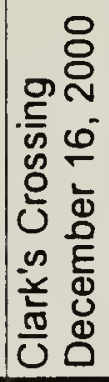 & 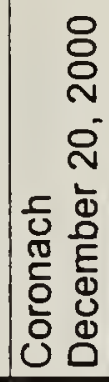 & 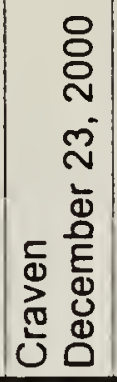 & 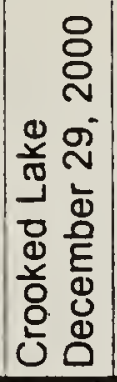 & 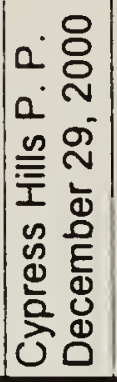 & 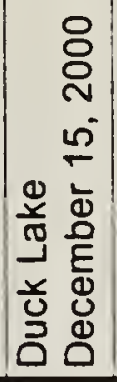 & SPECIES \\
\hline & & & & & 880 & & $\overrightarrow{17}$ & & & MALLARD \\
\hline & & & & 41 & 17 & & 1 & & & COMMON GOLDENEYE \\
\hline & & & & & 1 & & & & & BALD EAGLE \\
\hline & & & & 1 & & & 1 & & & NORTHERN GOSHAWK \\
\hline & & & & & 2 & 1 & & & & GOLDEN EAGLE \\
\hline 7 & 53 & 6 & & 177 & 31 & 11 & & & & GRAY PARTRIDGE \\
\hline & 22 & & & & 7 & & & & & RING-NECKED PHEASANT \\
\hline & & & 4 & & & & & & & SPRUCE GROUSE \\
\hline 1 & & & & 1 & & & & 1 & 2 & RUFFED GROUSE \\
\hline 1 & & 18 & & 32 & 13 & 16 & 3 & & & SHARP-TAILED GROUSE \\
\hline 42 & 18 & 0 & & 141 & 21 & 6 & 1 & & 35 & ROCK DOVE \\
\hline & 1 & 3 & & 7 & & & 1 & & & GREAT HORNED OWL \\
\hline & & 3 & & 3 & & 0 & & & & SNOWY OWL \\
\hline 1 & & & 3 & & & & & & 1 & GREAT GRAY OWL \\
\hline & & 2 & & & & & & & & SHORT-EARED OWL \\
\hline 5 & & & 1 & 6 & & 19 & 5 & 4 & & DOWNY WOODPECKER \\
\hline 2 & & & 3 & 11 & & 12 & 4 & 4 & $\overline{1}$ & HAIRY WOODPECKER \\
\hline & & & 1 & & & & & & & THREE-TOED WOODPECKER \\
\hline & & & 1 & & & & & & 1 & BLLACK-BACKED WOODPECKER \\
\hline & & & & & & 3 & & & & NORTHERN FLICKER (Y-s) \\
\hline & & & 2 & & & & & & 1 & PILEATED WOODPECKER \\
\hline & 2 & 66 & & & 11 & & & & & HORNED LARK \\
\hline & & & 13 & & & & & & & GRAY JAY \\
\hline 6 & & & 1 & & & 26 & 40 & 1 & & BLUE JAY \\
\hline 77 & 22 & 49 & 13 & 295 & 62 & 108 & 50 & 6 & 6 & BLACK-BILLED MAGPIE \\
\hline & & & & & & & & & & AMERICAN CROW \\
\hline 17 & & & 35 & 30 & & 8 & 12 & 19 & 13 & COMMON RAVEN \\
\hline 59 & & 0 & 23 & 153 & & 102 & 199 & 188 & 7 & BLACK-CAPPED CHICKADEE \\
\hline & & & 14 & & & & & & 6 & BOREAL CHICKADEE \\
\hline 1 & & & & 1 & & & & 60 & & RED-BREASTED NUTHATCH \\
\hline 2 & & & & & & 16 & 5 & 3 & & WHITE-BREASTED NUTHATCH \\
\hline & & & & 1 & & 0 & & & & AMERICAN ROBIN \\
\hline 42 & & 72 & & 9 & & 81 & 186 & & 162 & BOHEMIAN WAXWING \\
\hline & & & & & & 2 & & & & CEDAR WAXWING \\
\hline 0 & & & & & & 1 & & & & NORTHERN SHRIKE \\
\hline & 4 & & & 46 & 1 & & 3 & & & EUROPEAN STARLING \\
\hline 2 & & & & & & 1 & & & & HARRIS' SPARROW \\
\hline 1 & & & & 1 & & 1 & & & & DARK-EYED JUNCO (S-C) \\
\hline 14 & 75 & & & 2650 & 48 & & 5 & & & SNOW BUNTING \\
\hline & & & 1 & 10 & & & 2 & 15 & 3 & PINE GROSBEAK \\
\hline 0 & & & & & & 0 & & & & HOUSE FINCH \\
\hline & & & 1 & & & & 80 & & & COMMON REDPOLL \\
\hline & & & & 15 & & & & & & PINE SISKIN \\
\hline & & & & & & & & & & EVENING GROSBEAK \\
\hline 84 & 136 & 119 & & 1661 & 165 & 254 & 17 & & 22 & HOUSE SPARROW \\
\hline$\overline{364}$ & 337 & 388 & 116 & 5294 & 1268 & 676 & 632 & 307 & 260 & TOTAL INDIVIDUALS \\
\hline 18 & 11 & 10 & 15 & 23 & 19 & 19 & 19 & 7 & 13 & SPECIES ON COUNT DAY \\
\hline 20 & 11 & 12 & 15 & 23 & 19 & 24 & 19 & 7 & 13 & SPECIES COUNT PERIOD \\
\hline 0 & 4 & 50 & 0 & 2 & 9 & 8 & 0 & 6 & 0 & INDIVIDUALS IN TABLES $4 \& 5$ \\
\hline 0 & 2 & 1 & 0 & 2 & 6 & 3 & 0 & 1 & 0 & SPECIES IN TABLES $4 \& 5$ \\
\hline
\end{tabular}


Table 3-3. SPECIES RECORDED FROM NINE OR MORE LOCALITIES

$(0=$ a species recorded during count period but not seen on count day)

SPECIES
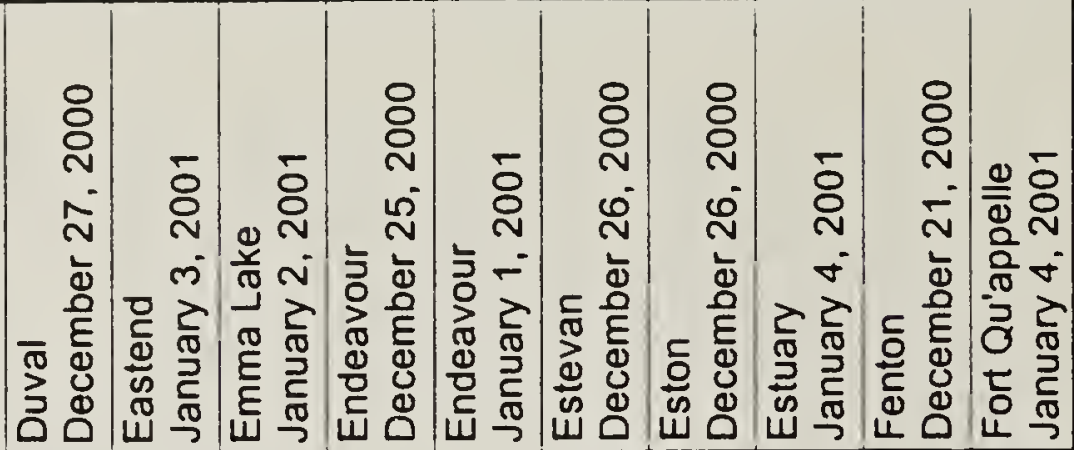

MALLARD

COMMON GOLDENEYE

BALD EAGLE

NORTHERN GOSHAWK

GOLDEN EAGLE

GRAY PARTRIDGE

RING-NECKED PHEASANT

SPRUCE GROUSE

RUFFED GROUSE

SHARP-TAILED GROUSE

ROCK DOVE

GREAT HORNED OWL

SNOWY OWL

GREAT GRAY OWL

SHORT-EARED OWL

DOWNY WOODPECKER

HAIRY WOODPECKER

THREE-TOED WOODPECKER

BLACK-BACKED WOODPECKER

NORTHERN FLICKER ( $Y$-s)

PILEATED WOODPECKER

HORNED LARK

GRAY JAY

BLUE JAY

BLACK-BILLED MAGPIE

AMERICAN CROW

COMMON RAVEN

BLACK-CAPPED CHICKADEE

BOREAL CHICKADEE

RED-BREASTED NUTHATCH

WHITE-BREASTED NUTHATCH

AMERICAN ROBIN

BOHEMIAN WAXWING

CEDAR WAXWING

NORTHERN SHRIKE

EUROPEAN STARLING

HARRIS' SPARROW

DARK-EYED JUNCO (S-C)

SNOW BUNTING

PINE GROSBEAK

HOUSE FINCH

COMMON REDPOLL

PINE SISKIN

EVENING GROSBEAK

HOUSE SPARROW

TOTAL INDIVIDUALS

SPECIES ON COUNT DAY

SPECIES COUNT PERIOD

INDIVIDUALS IN TABLES $4 \& 5$

SPECIES IN TABLES $4 \& 5$

\begin{tabular}{|c|c|c|c|c|c|c|c|c|}
\hline & & & & 500 & $\overline{1}$ & & & 40 \\
\hline & & & & 25 & & & & 1 \\
\hline & & & & & & & & \\
\hline & 1 & & & 1 & & 5 & & \\
\hline & 0 & & & & & & & \\
\hline 49 & & & & 17 & & & 20 & 0 \\
\hline & & & & 25 & 6 & 106 & & 0 \\
\hline & & & & & . & & & \\
\hline & & 4 & & & . & & 3 & 6 \\
\hline 22 & & & & 11 & 12 & 50 & 1 & 6 \\
\hline 42 & 6 & 3 & 8 & 49 & 1 & 27 & 0 & 16 \\
\hline & 0 & & & & 3 & 1 & $\overline{0}$ & 1 \\
\hline 1 & & & & 1 & 5 & & & \\
\hline
\end{tabular}

\begin{tabular}{|r|r|r|r|r|r|r|r|r|r|}
\hline & & & 1 & 5 & & & & & \\
\hline & & & & & & 7 & & & \\
\hline 1 & 1 & 1 & 1 & 1 & 5 & & 7 & 3 & 17 \\
\hline
\end{tabular}

R

\begin{tabular}{|l|l|l|l|l|l|l|l|l|l|}
\hline & & & & & & & & & \\
\hline & & 0 & & & & & & & \\
\hline
\end{tabular}

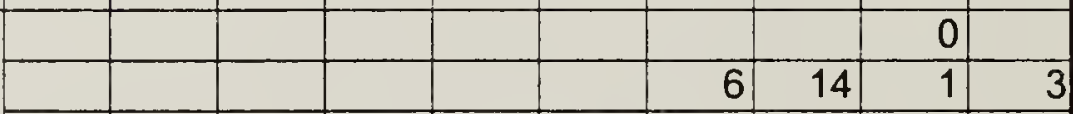

\begin{tabular}{|r|r|r|r|r|r|r|r|r|r|}
\hline & & & 1 & 4 & & & & & \\
\hline & 0 & 10 & 1 & 2 & 1 & 1 & 4 & 4 & 22 \\
\hline 29 & 14 & 2 & 11 & & 80 & 31 & 64 & 34 & 14 \\
\hline
\end{tabular}

\begin{tabular}{|r|r|r|r|r|r|r|r|r|r|}
\hline 29 & 14 & 2 & 11 & & 80 & 31 & 64 & 34 & 14 \\
\hline & & & & & & & & & \\
\hline 18 & 21 & 184 & 90 & 6 & & & & 16 & 24 \\
\hline & & 23 & 34 & 10 & 25 & 6 & 15 & 64 & 131 \\
\hline
\end{tabular}

H

\begin{tabular}{|r|r|r|r|r|r|r|r|r|r|}
\hline & & 7 & 2 & & 9 & & & & 10 \\
\hline 66 & & & & & 1 & & & 0 & 0 \\
\hline & 36 & & & & 63 & 0 & 87 & 166 & 310 \\
\hline
\end{tabular}


Table 3-4. SPECIES RECORDED FROM NINE OR MORE LOCALITIES

$(0=a$ species recorded duning count period but not seen on count day)

\begin{tabular}{|c|c|c|c|c|c|c|c|c|c|c|}
\hline 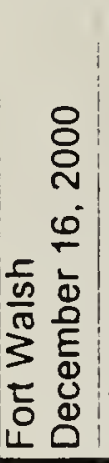 & 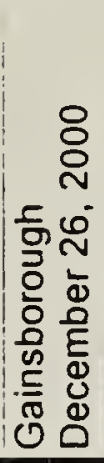 & 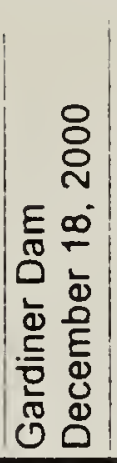 & 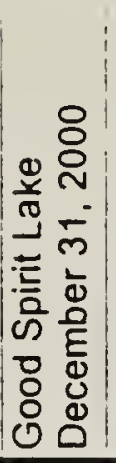 & 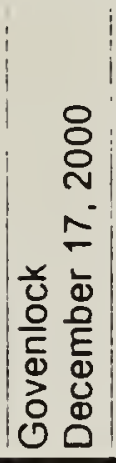 & 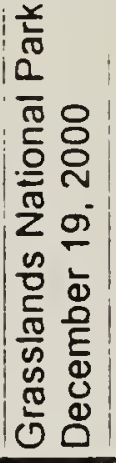 & 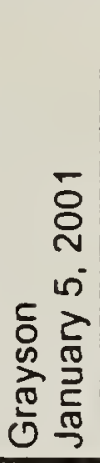 & 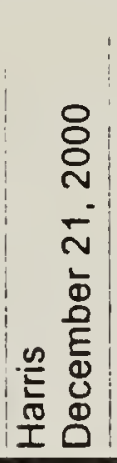 & 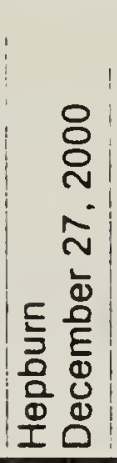 & 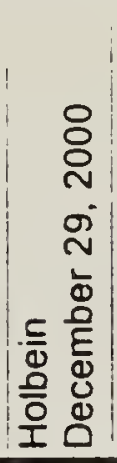 & SPECIES \\
\hline 1 & & 1840 & & 3 & & & & & & MALLARD \\
\hline 7 & & 60 & & & & & & & & COMMON GOLDENEYE \\
\hline 3 & & 23 & & & 1 & & & & & BALD EAGLE \\
\hline 1 & & & & & & & & & & INORTHERN GOSHAWK \\
\hline & & 2 & & 1 & & & & & & GOLDEN EAGLE \\
\hline 4 & 15 & 117 & 15 & 29 & 38 & & 4 & & & GRAY PARTRIDGE \\
\hline 2 & 3 & & & 28 & 2 & & & & & IRING-NECKED PHEASANT \\
\hline & & & & i & & & & & & ISPRUCE GROUSE \\
\hline 0 & & 1 & 1 & & & & & & & RUFFED GROUSE \\
\hline 10 & 5 & 16 & & $8 !$ & 78 & & $80 !$ & & & SHARP-TAILED GROUSE \\
\hline & 27 & 58 & 4 & & 6 & & 115 & & & ROCK DOVE \\
\hline 5 & 2 & 4 & & 7 & 1 & & 11 & & & IGREAT HORNED OWL \\
\hline 11 & 1 & 4 & & 2 & & & & 1 & 1 & ISNOWY OWL \\
\hline & & & 1 & & & & & 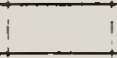 & 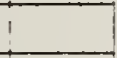 & TGRAT GRAY OWL \\
\hline & & 3 & 1 & 3 & & & 1 & & & TSHORT-EARED OWL \\
\hline 2 & 1 & & 6 & & & 5 & 1 & 0 & 21 & DOWNY WOODPECKER \\
\hline 3 & & 1 & 4 & & & 2 & 3 & 0 & 2 & HAIRY WOODPECKER \\
\hline $1:$ & & $i$ & i & & & & & 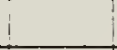 & & THREE-TOED WOODPECKER \\
\hline & & & & & & & & & & BLACK-BACKED WOODPECKER \\
\hline & & & & & $!$ & & & 1 & 1 & NORTHERN FLICKER (Y-s) \\
\hline & & $T$ & 0 & & i & & & & i & IPILEATED WOODPECKER \\
\hline & 40 & $\mid 1$ & & 287 & 180 & & & & & HORNED LARK \\
\hline 7 & & & & & 7 & & & & & IGRAY JAY \\
\hline t & 1 & 1 & $3 !$ & & & & & 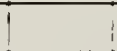 & 6 & BLUE JAY \\
\hline 75 & 23 & 246 & $42 !$ & 26 & 34 & 2 & 144 & 1 & 21 & BLACK-BILLED MAGPIE \\
\hline 1 & & & & & & & & & & AMERICAN CROW \\
\hline 6 & 2 & 12 & 16 & & & 2 & 11 & 1 & 1 & COMMON RAVEN \\
\hline 26 & 4 & 28 & 36 & & 4 & 24 & 25 & 5 & 8 & BLACK-CAPPED CHICKADEE \\
\hline & & & & & & & & & & BOREAL CHICKADEE \\
\hline 2 & & & & & & & & & & RED-BREASTED NUTHATCH \\
\hline & & & 6 & & & 2 & & & & WHITE-BREASTED NUTHATCH \\
\hline 1 & & & & 1 & & & & 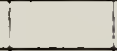 & & AMERICAN ROBIN \\
\hline 2 & 100 & 25 & 1 & T & & 18 & 54 & & & BOHEMIAN WAXWING \\
\hline & & & & & & & & & & CEDAR WAXWING \\
\hline 1 & & & & & & & & & $i$ & NORTHERN SHRIKE \\
\hline 2 & & 8 & 2 & 12 & & & 2 & & & EUROPEAN STARLING \\
\hline 3 & & & & & & & & & I & HARRIS' SPARROW \\
\hline & & & & & & & & & & DARK-EYED JUNCO (S-C) \\
\hline 20 & 75 & 190 & 33 & 1540 & $\mid 609$ & & 140 & & & TSNOW BUNTING \\
\hline & & 5 & 20 & & & & & & 6 & PINE GROSBEAK \\
\hline & & & & & & & & & & HOUSE FINCH \\
\hline 2 & & & & & 15 & & 6 & & & COMMON REDPOLL \\
\hline & & & 2. & & & & & & & PINE SISKIN \\
\hline & & & 0 & & & & & & & EVENING GROSBEAK \\
\hline 112 & 57 & 11743 & 39 & 208 & 56 & 30 & 226 & 10 & 25 & 5 HOUSE SPARROW \\
\hline$\overline{348}$ & 379 & 4693 & 231 & 2164 & 1024 & $\overline{85}$ & 815 & 17 & 52 & 2ITOTAL INDIVIDUALS \\
\hline 311 & 16 & 25 & 17 & 18 & 12 & 8 & 17 & 4 & 8 & SPECIES ON COUNT DAY \\
\hline 32 & 16 & 25 & 20 & 18 & 12 & 8 & 17 & 6 & 8 & SPECIES COUNT PERIOD \\
\hline 64 & 23 & 307 & $\overrightarrow{0}$ & 10 & 0 & 0 & 2 & 0 & 0 & IINDIVIDUALS IN TABLES $4 \& 5$ \\
\hline 9 & 1 & 5 & 1 & 5 & 0 & 0 & 2 & 0 & 0 & TSPECIES IN TABLES $4 \& 5$ \\
\hline
\end{tabular}


Table 3-5. SPECIES RECORDED FROM NINE OR MORE LOCALITIES

$(0=a$ species recorded during count period but not seen on count day)

SPECIES

MALLARD

COMMON GOLDENEYE

BALD EAGLE

NORTHERN GOSHAWK

GOLDEN EAGLE

GRAY PARTRIDGE

RING-NECKED PHEASANT

SPRUCE GROUSE

RUFFED GROUSE

SHARP-TAILED GROUSE

ROCK DOVE

GREAT HORNED OWL

SNOWY OWL

GREAT GRAY OWL

SHORT-EARED OWL

DOWNY WOODPECKER

HAIRY WOODPECKER

THREE-TOED WOODPECKER

BLACK-BACKED WOODPECKER

NORTHERN FLICKER (Y-s)

PILEATED WOODPECKER

HORNED LARK

GRAY JAY

BLUE JAY

BLACK-BILLED MAGPIE

AMERICAN CROW

COMMON RAVEN

BLACK-CAPPED CHICKADEE

BOREAL CHICKADEE

RED-BREASTED NUTHATCH

WHITE-BREASTED NUTHATCH

AMERICAN ROBIN

BOHEMIAN WAXWING

CEDAR WAXWING

NORTHERN SHRIKE

EUROPEAN STARLING

HARRIS' SPARROW

DARK-EYED JUNCO (S-C)

SNOW BUNTING

PINE GROSBEAK

HOUSE FINCH

COMMON REDPOLL

PINE SISKIN

EVENING GROSBEAK

HOUSE SPARROW

TOTAL INDIVIDUALS

SPECIES ON COUNT DAY

SPECIES COUNT PERIOD

INDIVIDUALS IN TABLES $4 \& 5$

SPECIES IN TABLES $4 \& 5$

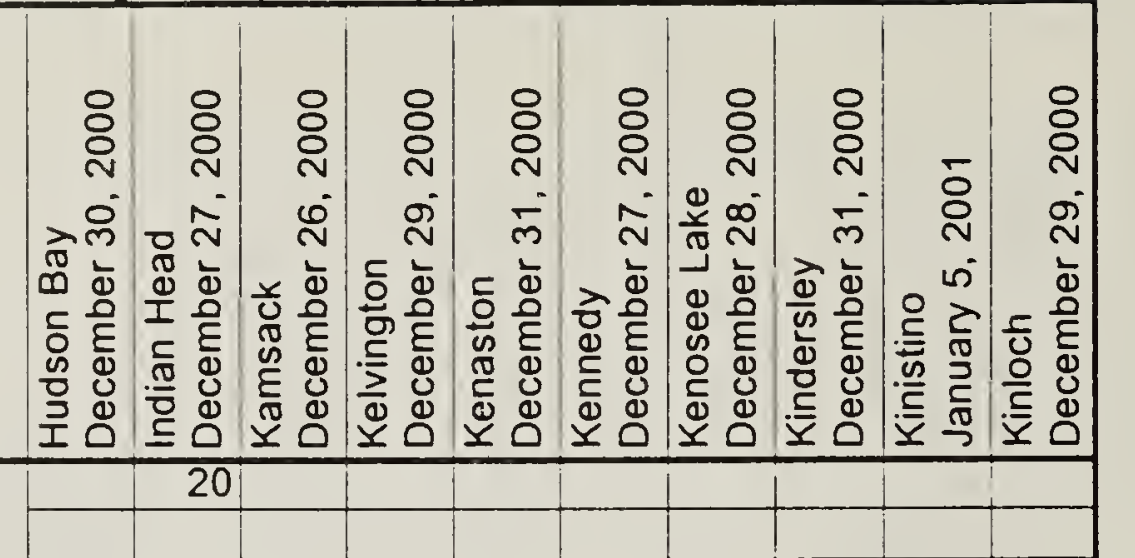

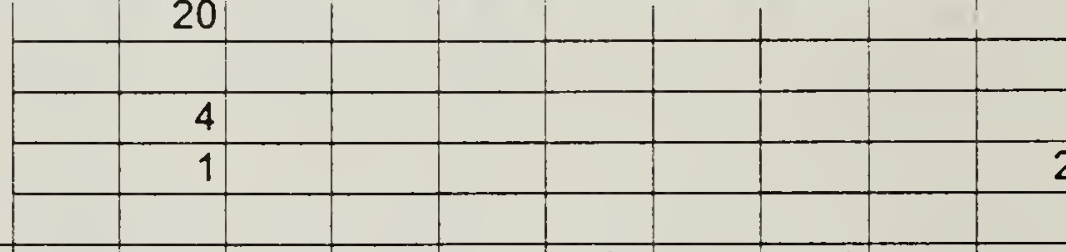

\begin{tabular}{|r|r|r|r|r|r|r|r|r|r|}
\hline & 11 & 0 & & 25 & 20 & & 9 & \\
\hline & & & & & 1 & & & \\
\hline & 2 & & & & & & & & \\
\hline & & & 0 & & & & & & 0 \\
\hline
\end{tabular}

\begin{tabular}{|r|r|r|r|r|r|r|r|r|r|}
\hline & 8 & 10 & & 0 & 14 & & & & \\
\hline 2 & 10 & 16 & & 112 & 19 & & 55 & 12 & 17 \\
\hline & 1 & & & & & & & & 0 \\
\hline
\end{tabular}

\begin{tabular}{|r|r|r|r|r|r|r|r|r|r|}
\hline & 1 & & & & & & & 0 \\
\hline 3 & 1 & 2 & & & 1 & 1 & 1 & 0 \\
\hline & & & & & & & & & 4 \\
\hline
\end{tabular}

\begin{tabular}{|r|r|r|r|r|r|r|r|r|r|}
\hline 1 & 14 & 19 & 4 & & 15 & 7 & & & 8 \\
\hline 4 & 7 & 19 & 2 & & 13 & 17 & & 1 & 6 \\
\hline 2 & & & & & & & & & \\
\hline
\end{tabular}

\begin{tabular}{|r|r|r|r|r|r|r|r|r|}
\hline & & & & & & & & \\
\hline & 2 & 1 & & & 1 & & & 0 \\
\hline
\end{tabular}

\begin{tabular}{|r|r|r|r|r|r|r|r|r|r|}
\hline & & & & 9 & & & & & \\
\hline 17 & & 2 & & & & & & & 9 \\
\hline 27 & 12 & 100 & 2 & & 9 & 44 & & & 25 \\
\hline 9 & 32 & 19 & 15 & 42 & 54 & 13 & 17 & 3 & 36 \\
\hline
\end{tabular}

\begin{tabular}{|r|r|r|r|r|r|r|r|r|r|}
\hline 12 & 5 & 34 & 7 & 0 & 7 & 12 & 0 & 2 & 28 \\
\hline 49 & 88 & 134 & 20 & 0 & 48 & 193 & 2 & 12 & 74 \\
\hline & & 3 & & & & & & & 0 \\
\hline
\end{tabular}


Table 3-6. SPECIES RECORDED FROM NINE OR MORE LOCALITIES

$(0=$ a species recorded during count period but not seen on count day)

\begin{tabular}{|c|c|c|c|c|c|c|c|c|c|c|}
\hline 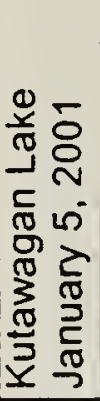 & 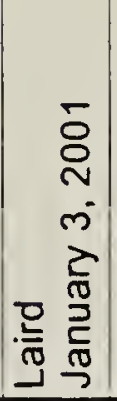 & 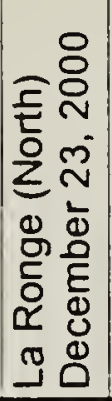 & 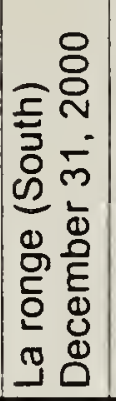 & 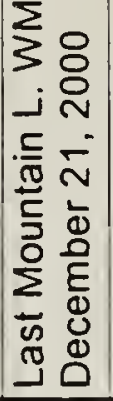 & 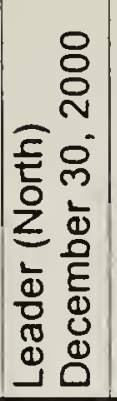 & 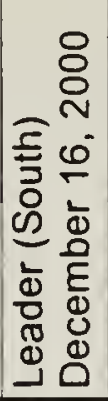 & 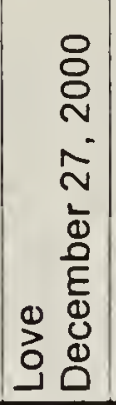 & 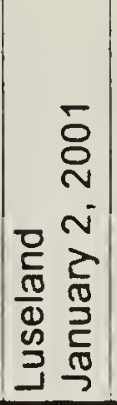 & 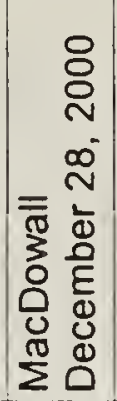 & SPECIES \\
\hline & & & & & & & i & 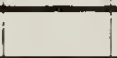 & & MALLARD \\
\hline & & & & & & & & & & COMMON GOLDENEYE \\
\hline & & & & 0 & & 1 & & & 0 & BALD EAGLE \\
\hline & & & & & & & 1 & 1 & & NORTHERN GOSHAWK \\
\hline & & & & & 1 & 1 & & & & GOLDEN EAGLE \\
\hline 47 & 0 & & & 111 & 16 & & & 20 & & GRAY PARTRIDGE \\
\hline & & & & & 34 & 8 & & & & RING-NECKED PHEASANT \\
\hline & & 1 & 1 & & & & 0) & & 2 & SPRUCE GROUSE \\
\hline & & & & & & & 0 & & 2 & RUFFED GROUSE \\
\hline 13 & & & & 37 & & 6 & & & 12 & SHARP-TAILED GROUSE \\
\hline & & & & 227 & 16 & 4 & 3 & 14 & 80 & ROCK DOVE \\
\hline & & & & 1 & 1 & 2 & & 2 & $\overline{1}$ & GREAT HORNED OWL \\
\hline 1 & 0 & & & 3 & & 2 & & 1 & 1 & SNOWY OWL \\
\hline & & & & & & & 12 & & $\overline{0}$ & GREAT GRAY OWL \\
\hline & & & & & 1 & 5 & & & & SHORT-EARED OWL \\
\hline & 2 & & 1 & & 1 & 3 & 6 & 2 & 4 & DOWNY WOODPECKER \\
\hline & 3 & 2 & 4 & & & 2 & 7 & 3 & 4 & HAIRY WOODPECKER \\
\hline & & & & & & & & & & THREE-TOED WOODPECKER \\
\hline & & & 1 & & & & 1 & & & BLACK-BACKED WOODPECKER \\
\hline & & & & & & 1 & & & & NORTHERN FLICKER (Y-s) \\
\hline & & & 0 & & 1 & & & & 0 & PILEATED WOODPECKER \\
\hline 2 & & & & & & 104 & & & & HORNED LARK \\
\hline & & 1 & 17 & & & & 4 & & 6 & GRAY JAY \\
\hline & & & 2 & & 1 & & 11 & 9 & 6 & BLUE JAY \\
\hline 24 & 12 & & & 96 & 35 & 52 & 26 & 42 & 8 & BLACK-BILLED MAGPIE \\
\hline & & & & & & & & & & AMERICAN CROW \\
\hline 2 & 1 & 37 & 100 & & & & 64 & 1 & 30 & COMMON RAVEN \\
\hline 2 & 7 & 15 & 25 & 6 & 1 & 5 & 64 & 41 & 60 & BLACK-CAPPED CHICKADEE \\
\hline & & 0 & 12 & & & & 2 & & & BOREAL CHICKADEE \\
\hline & 0 & & 5 & & & 1 & 2 & & & RED-BREASTED NUTHATCH \\
\hline & & & & & & 1 & 2 & & & WHITE-BREASTED NUTHATCH \\
\hline & & & & & & & & 0 & & AMERICAN ROBIN \\
\hline & 0 & & & & 10 & & 7 & 127 & 20 & BOHEMIAN WAXWING \\
\hline & & & & & & & & 1 & & CEDAR WAXWING \\
\hline & & & & & & & & & 1 & NORTHERN SHRIKE \\
\hline & & 3 & & & & 20 & 1 & & & EUROPEAN STARLING \\
\hline & & & & & & & & & & HARRIS' SPARROW \\
\hline & & & & & & & & & & DARK-EYED JUNCO (S-C) \\
\hline 75 & & & & 522 & & 2 & 1260 & 125 & 100 & SNOW BUNTING \\
\hline & & 12 & 11 & & & & 77 & & 50 & PINE GROSBEAK \\
\hline & & & & & & 14 & & & & HOUSE FINCH \\
\hline & & & 6 & & & & 178 & & & COMMON REDPOLL \\
\hline & & & & & & & & & 20 & PINE SISKIN \\
\hline & & & 13 & & & & 168 & & 20 & EVENING GROSBEAK \\
\hline 53 & 20 & & & 530 & & 300 & 18 & 74 & & HOUSE SPARROW \\
\hline$\overline{219}$ & 45 & $\overline{73}$ & 197 & $\sqrt{1533}$ & 119 & 538 & 1919 & 463 & 427 & TOTAL INDIVIDUALS \\
\hline$\overline{9}$ & 6 & 7 & 13 & 9 & 13 & 24 & 22 & 15 & 19 & SPECIES ON COUNT DAY \\
\hline 9 & 10 & 8 & 14 & 10 & 13 & $\overline{24}$ & 24 & 17 & 22 & SPECIES COUNT PERIOD \\
\hline 0 & 0 & 2 & 0 & $\overline{0}$ & 1 & 4 & 5 & 0 & 0 & INDIVIDUALS IN TABLES $4 \& 5$ \\
\hline 0 & 0 & 1 & 0 & 0 & 1 & 4 & 1 & 1 & 0 & SPECIES IN TABLES $4 \& 5$ \\
\hline
\end{tabular}


Table 3-7. SPECIES RECORDED FROM NINE OR MORE LOCALITIES

$(0=$ a species recorded during count period but not seen on count day)

SPECIES

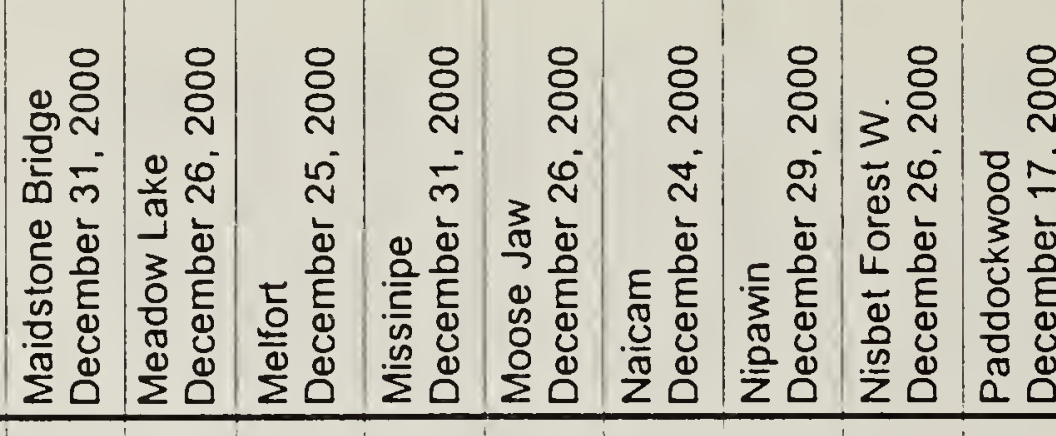

MALLARD

COMMON GOLDENEYE

BALD EAGLE

NORTHERN GOSHAWK

GOLDEN EAGLE

GRAY PARTRIDGE

RING-NECKED PHEASANT

SPRUCE GROUSE

RUFFED GROUSE

SHARP-TAILED GROUSE

ROCK DOVE

GREAT HORNED OWL

SNOWY OWL

GREAT GRAY OWL

SHORT-EARED OWL

DOWNY WOODPECKER

HAIRY WOODPECKER

THREE-TOED WOODPECKER

BLACK-BACKED WOODPECKER

NORTHERN FLICKER (Y-s)

PILEATED WOODPECKER

HORNED LARK

GRAY JAY

BLUE JAY

BLACK-BILLED MAGPIE

AMERICAN CROW

COMMON RAVEN

BLACK-CAPPED CHICKADEE

BOREAL CHICKADEE

RED-BREASTED NUTHATCH

WHITE-BREASTED NUTHATCH

AMERICAN ROBIN

BOHEMIAN WAXWING

CEDAR WAXWING

NORTHERN SHRIKE

EUROPEAN STARLING

HARRIS' SPARROW

DARK-EYED JUNCO (S-C)

SNOW BUNTING

PINE GROSBEAK

HOUSE FINCH

COMMON REDPOLL

PINE SISKIN

EVENING GROSBEAK

HOUSE SPARROW

TOTAL INDIVIDUALS

SPECIES ON COUNT DAY

SPECIES COUNT PERIOD

INDIVIDUALS IN TABLES $4 \& 5$

SPECIES IN TABLES 4\&5

\begin{tabular}{|c|c|c|c|c|c|c|c|c|}
\hline & & & & & & & & \\
\hline & & & & & & & 0 & \\
\hline & & & & & & & 1 & \\
\hline & & & & & & & & \\
\hline & & & & 64 & 15 & & 4 & \\
\hline & & & & 10 & & & & \\
\hline & & & & & & & & \\
\hline 1 & 1 & & 1 & & & 2 & 1 & 1 \\
\hline 3 & & & & & & & & \\
\hline & 8 & & & 367 & 0 & & 4 & \\
\hline 7 & & & & 1 & & & & \\
\hline 1 & & & & 3 & & & & \\
\hline 1 & 2 & & & & 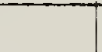 & 3 & 1 & 2 \\
\hline 1 & & & & & & & & \\
\hline 1 & 3 & 1 & 2 & 13 & 0 & 0 & 3 & \\
\hline 2 & 4 & & 1 & 3 & 1 & 0 & 3 & 1 \\
\hline & & & & & & 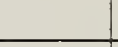 & -1 & \\
\hline & 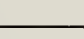 & 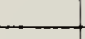 & - & & & - & & \\
\hline & & & & 6 & & & & \\
\hline & 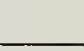 & & & & & 1 & 1 & \\
\hline & 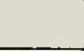 & & & 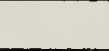 & 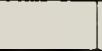 & & 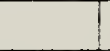 & \\
\hline & 6 & & 8 & 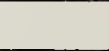 & & 0 & 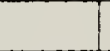 & 1 \\
\hline 2 & 4 & & 1 & 4 & 2 & 3 & 17 & 1 \\
\hline 42 & 10 & 2 & & 54 & 13 & 25 & 11 & 1 \\
\hline & & & & 4 & & & & \\
\hline 33 & 54 & 2 & 28 & & 12 & 92 & 25 & 1 \\
\hline 10 & 84 & 1 & 12 & 36 & 21 & 21 & 23 & 30 \\
\hline & & & 6 & & & & 41 & \\
\hline & 1 & & & 18 & & 4 & & \\
\hline & & & & 8 & & & 2 & 1 \\
\hline & & & & & & & 1 & \\
\hline 6 & & 1 & & & 11 & 8 & & \\
\hline & & & & & & & & \\
\hline & & & & & & & & \\
\hline 1 & & & & & & & & \\
\hline & & & & & & & & \\
\hline & & & 1 & 16 & & & & \\
\hline 160 & 250 & & & & 345 & 1465 & 680 & \\
\hline 7 & 42 & 1 & 22 & & 2 & 8 & 39 & 12 \\
\hline & & & & 20 & & & & \\
\hline & 30 & & 46 & & & & 0 & 7 \\
\hline & & & & 3 & & 1 & 9 & \\
\hline & 14 & & & & & $\mid 48$ & 90 & 7 \\
\hline 30 & & 3 & & 400 & 135 & 25 & & $\overline{7}$ \\
\hline 309 & 514 & $\overline{11 \mid}$ & 128 & 1032 & 557 & 1710 & 918 & 72 \\
\hline 18 & $\overline{16}$ & 7 & 11 & 19 & 10 & 15 & 18 & 13 \\
\hline 18 & 16 & 7 & 11 & 23 & 12 & 18 & 22 & 13 \\
\hline 1 & 1 & 0 & 0 & 2 & 0 & 4 & 0 & 0 \\
\hline 1 & 1 & 0 & 0 & 5 & 0 & 1 & 2 & 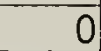 \\
\hline
\end{tabular}


Table 3-8. SPECIES RECORDED FROM NINE OR MORE LOCALITIES $(0=$ a species recorded during count period but not seen on count day)

\begin{tabular}{|c|c|c|c|c|c|c|c|c|c|}
\hline 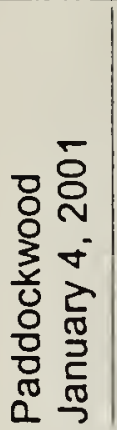 & 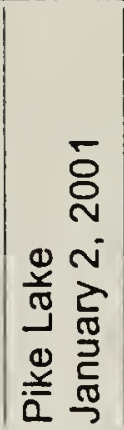 & 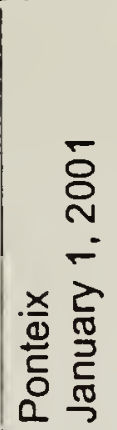 & 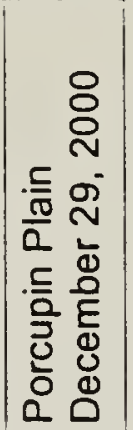 & 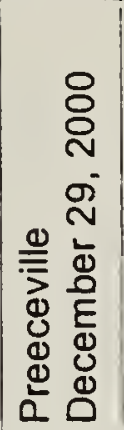 & 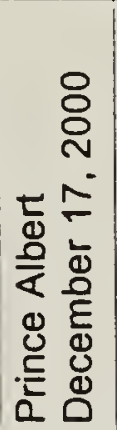 & 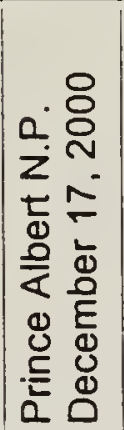 & 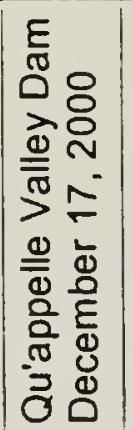 & 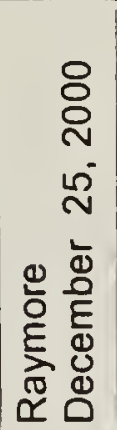 & SPECIES \\
\hline & & & & & & & 11 & & MALLARD \\
\hline & & & & & & & 6 & & COMMON GOLDENEYE \\
\hline & 1 & & & & & & & & BALD EAGLE \\
\hline & 1 & & & & & & & & NORTHERN GOSHAWK \\
\hline & 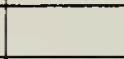 & & & & & & & & GOLDEN EAGLE \\
\hline & 3 & & & & & & 8 & 6 & GRAY PARTRIDGE \\
\hline & & 2 & & & & & 1 & & RING-NECKED PHEASANT \\
\hline & & & & & & & & & SPRUCE GROUSE \\
\hline & 3 & & & 3 & & 5 & & 2 & RUFFED GROUSE \\
\hline 2 & 103 & & & 1 & & & 15 & 10 & SHARP-TAILED GROUSE \\
\hline & 86 & 12 & & 21 & 102 & & 23 & 21 & ROCK DOVE \\
\hline & & & & 1 & & & 1 & $\overline{2}$ & GREAT HORNED OWL \\
\hline & & & & & & & & & SNOWY OWL \\
\hline 6 & & & & 1 & 2 & & & & GREAT GRAY OWL \\
\hline & & & & & & & 1 & & SHORT-EARED OWL \\
\hline 2 & 17 & & 1 & 18 & 4 & & & 1 & DOWNY WOODPECKER \\
\hline 2 & 22 & & & 13 & 3 & 2 & & 1 & HAIRY WOODPECKER \\
\hline & & & & & & & & & THREE-TOED WOODPECKER \\
\hline & & & & & & 1 & & & BLACK-BACKED WOODPECKER \\
\hline & 1 & & & & & & & & NORTHERN FLICKER (Y-s) \\
\hline & 2 & & & 5 & & & & & PILEATED WOODPECKER \\
\hline & 1 & & & & & & 17 & 3 & HORNED LARK \\
\hline 4 & & & & 6 & 4 & 4 & & & GRAY JAY \\
\hline 8 & 41 & & 3. & 12 & 4 & & 1 & & BLUE JAY \\
\hline 5 & 208 & 6 & & 44 & 44 & 2 & 40 & 63 & BLACK-BILLED MAGPIE \\
\hline & 3 & & & & & & & & AMERICAN CROW \\
\hline 35 & 61 & & & 36 & 81 & 5 & 1 & 29 & COMMON RAVEN \\
\hline 14 & 346 & 1 & 7 & 37 & 56 & 16 & 10 & 15 & BLACK-CAPPED CHICKADEE \\
\hline & & & & 4 & & & & & BOREAL CHICKADEE \\
\hline & & & & 2 & & & & & RED-BREASTED NUTHATCH \\
\hline 1 & 7 & & 1 & & & & & & WHITE-BREASTED NUTHATCH \\
\hline & 7 & & & & 1 & & & & AMERICAN ROBIN \\
\hline & 1039 & & 0 & & 8080 & & & 79 & BOHEMIAN WAXWING \\
\hline & & & & & & & & & CEDAR WAXWING \\
\hline & 3 & & & 1 & & & & 0 & NORTHERN SHRIKE \\
\hline & & & & & & & & 5 & EUROPEAN STARLING \\
\hline & & 1 & & & & & & & HARRIS' SPARROW \\
\hline & & & & & 1 & & & & DARK-EYED JUNCO (S-C) \\
\hline 200 & 165 & & 0 & 111 & 575 & & 588 & 37 & SNOW BUNTING \\
\hline 55 & 32 & & 7 & 91 & 34 & 4 & & 9 & PINE GROSBEAK \\
\hline & & 6 & & & & & & & HOUSE FINCH \\
\hline 8 & 35 & & & 0 & & & & & COMMON REDPOLL \\
\hline & & & & $\overrightarrow{0}$ & 25 & & & & PINE SISKIN \\
\hline 23 & & & 23 & 131 & 13 & & & & EVENING GROSBEAK \\
\hline 12 & 212 & 20 & 10 & 38 & 113 & & 312 & 82 & HOUSE SPARROW \\
\hline 377 & 2403 & $\overline{49}$ & 52 & 576 & 9152 & $\overline{39}$ & 1027 & 366 & TOTAL INDIVIDUALS \\
\hline 15 & 27 & 8 & 7 & 20 & 21 & 8 & 17 & 17 & SPECIES ON COUNT DAY \\
\hline 15 & 28 & 8 & 9 & 24 & 21 & 8 & 17 & 18 & SPECIES COUNT PERIOD \\
\hline 0 & 4 & 1 & 0 & 0 & 10 & 0 & 2 & 0 & INDIVIDUALS IN TABLES $4 \& 5$ \\
\hline 0 & 4 & 1 & 0 & 2 & 4 & 0 & 2 & 0 & SPECIES IN TABLES $4 \& 5$ \\
\hline
\end{tabular}


Table 3-9. SPECIES RECORDED FROM NINE OR MORE LOCALITIES

$(0=$ a species recorded during count period but not seen on count day)

SPECIES

MALLARD

COMMON GOLDENEYE

BALD EAGLE

NORTHERN GOSHAWK

GOLDEN EAGLE

GRAY PARTRIDGE

RING-NECKED PHEASANT

SPRUCE GROUSE

RUFFED GROUSE

SHARP-TAILED GROUSE

ROCK DOVE

GREAT HORNED OWL

SNOWY OWL

GREAT GRAY OWL

SHORT-EARED OWL

DOWNY WOODPECKER

HAIRY WOODPECKER

THREE-TOED WOODPECKER

BLACK-BACKED WOODPECKER

NORTHERN FLICKER (Y-s)

PILEATED WOODPECKER

HORNED LARK

GRAY JAY

BLUE JAY

BLACK-BILLED MAGPIE

AMERICAN CROW

COMMON RAVEN

BLACK-CAPPED CHICKADEE

BOREAL CHICKADEE

RED-BREASTED NUTHATCH

WHITE-BREASTED NUTHATCH

AMERICAN ROBIN

BOHEMIAN WAXWING

CEDAR WAXWING

NORTHERN SHRIKE

EUROPEAN STARLING

HARRIS' SPARROW

DARK-EYED JUNCO (S-C)

SNOW BUNTING

PINE GROSBEAK

HOUSE FINCH

COMMON REDPOLL

PINE SISKIN

EVENING GROSBEAK

HOUSE SPARROW

TOTAL INDIVIDUALS

SPECIES ON COUNT DAY

SPECIES COUNT PERIOD

INDIVIDUALS IN TABLES $4 \& 5$

SPECIES IN TABLES $4 \& 5$

\begin{tabular}{|c|c|c|c|c|c|c|c|c|}
\hline 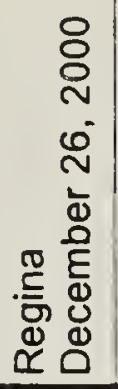 & 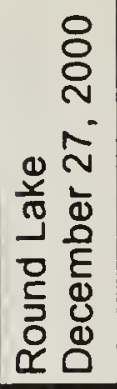 & 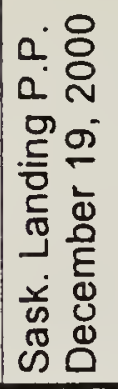 & 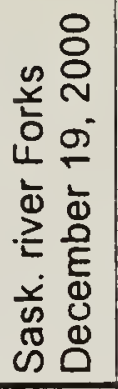 & 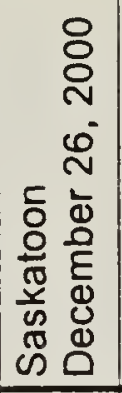 & 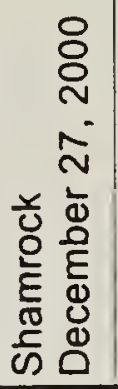 & 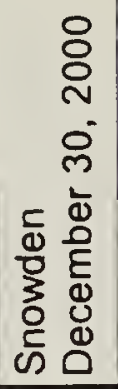 & 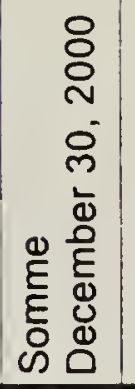 & 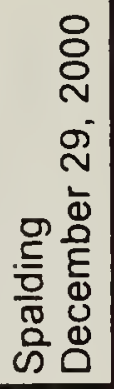 \\
\hline \multirow[t]{5}{*}{44} & 33 & 100 & & 5 & & & & \\
\hline & 4 & & & 234 & & & & \\
\hline & & & & & & & & \\
\hline & 1 & & & 1 & & & & \\
\hline & $\overline{2}$ & 2 & & & 1 & & & \\
\hline 135 & 6 & 73 & & 40 & 52 & & 0 & \\
\hline \multirow[t]{2}{*}{1} & & 0 & & 0 & 3 & & & \\
\hline & & & & & & 1 & & \\
\hline 1 & 1 & & 1 & & & 6 & 2 & \\
\hline 28 & 2 & 34 & & 67 & 54 & 4 & 19 & \\
\hline 1064 & 20 & 24 & 3 & 2472 & 10 & & 11 & 10 \\
\hline 5 & 1 & 1 & & 3 & 4 & & 0 & \\
\hline \multirow[t]{2}{*}{9} & & & & 0 & 3 & & & \\
\hline & & & 1 & & & 18 & 5 & \\
\hline 1 & & 1 & & 1 & & & & \\
\hline 13 & 12 & 2 & 1 & 46 & & 11 & 3 & 2 \\
\hline \multirow[t]{3}{*}{6} & 10 & 1 & 2 & 22 & & 8 & 5 & 1 \\
\hline & & & 1 & & & & 1 & \\
\hline & & & 2 & & & & & \\
\hline \multirow[t]{4}{*}{5} & 1 & & & 14 & & & & \\
\hline & 1 & & 1 & & & & 0 & \\
\hline & & 32 & & & 50 & & & \\
\hline & & & & & & 9 & 5 & \\
\hline 4 & 36 & & 4 & 63 & & 15 & 14 & \\
\hline 177 & 50 & 63 & 22 & 732 & 24 & 45 & 28 & 5 \\
\hline 4 & & & & 10 & & & & \\
\hline 5 & 19 & & 16 & 48 & & 190 & 48 & 4 \\
\hline \multirow[t]{2}{*}{40} & 105 & 13 & 30 & 732 & & 70 & 47 & 14 \\
\hline & & & 5 & & & 9 & & \\
\hline 18 & & & & 73 & & 3 & & \\
\hline 6 & 8 & & 2 & 5 & & 2 & 2 & \\
\hline \multirow[t]{4}{*}{0} & & & & 5 & & & & \\
\hline & 327 & 20 & & 311 & 4 & & 5 & \\
\hline & & & & 114 & & & & \\
\hline & & & & & & & & \\
\hline \multirow[t]{2}{*}{229} & & & & 5 & 6 & 5 & & \\
\hline & & & & & & & & \\
\hline 18 & & & & 9 & 1 & & 3 & \\
\hline \multirow[t]{2}{*}{59} & & 4 & & 450 & 21 & 1052 & 25 & \\
\hline & 38 & & 14 & 22 & & 155 & 19 & 1 \\
\hline \multirow[t]{4}{*}{103} & & & & 192 & & & & \\
\hline & & & 1 & 15 & & 53 & 12 & \\
\hline & & & & 123 & & 10 & & \\
\hline & 0 & & 3 & & & 130 & 65 & \\
\hline 679 & 35 & 140 & 22 & 4110 & 233 & 56 & 68 & 45 \\
\hline 2980 & 716 & $\overline{513}$ & 132 & 9951 & 468 & 1853 & 387 & 82 \\
\hline 31 & 23 & 18 & 19 & 42 & 15 & 22 & 20 & 8 \\
\hline 32 & 24 & 19 & 19 & 45 & 15 & 22 & 25 & $\overline{9}$ \\
\hline 326 & 4 & 3 & 1 & 27 & 2 & 1 & 0 & 0 \\
\hline 8 & 2 & 3 & 1 & 14 & 1 & 1 & 2 & - \\
\hline
\end{tabular}


Table 3-10. SPECIES RECORDED FROM NINE OR MORE LOCALITIES

$(0=$ a species recorded during count period but not seen on count day)

\begin{tabular}{|c|c|c|c|c|c|c|c|c|c|}
\hline 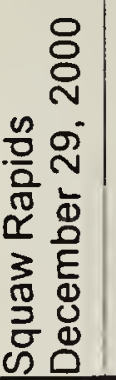 & 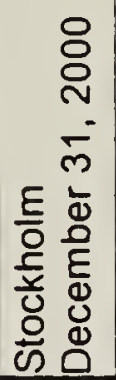 & 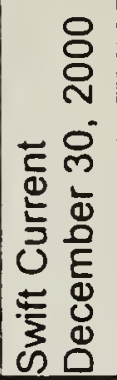 & 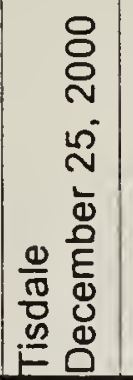 & 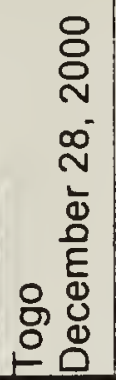 & 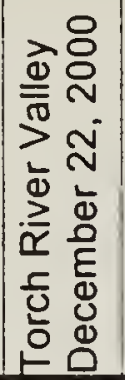 & 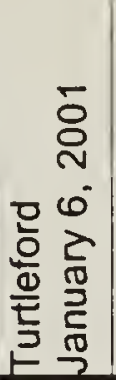 & 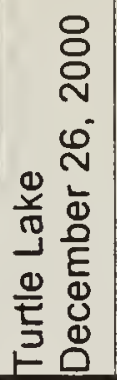 & 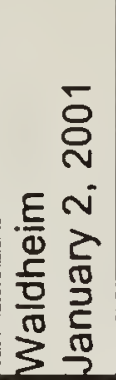 & SPECIES \\
\hline 3 & 12 & 3 & & & & & & & MALLARD \\
\hline 123 & & & & & & & & & COMMON GOLDENEYE \\
\hline 5 & 0 & & & 1 & & & & & BALD EAGLE \\
\hline & & & & & & & & & NORTHERN GOSHAWK \\
\hline & & 1 & & & & & & & GOLDEN EAGLE \\
\hline & 6 & 85 & & & & & & & GRAY PARTRIDGE \\
\hline & & 4 & & & & & & & RING-NECKED PHEASANT \\
\hline & & & & & & & 9 & & SPRUCE GROUSE \\
\hline & 1 & & & & & 2 & 8 & & RUFFED GROUSE \\
\hline 25 & 6 & 5 & & 0 & 2 & 7 & 2 & & SHARP-TAILED GROUSE \\
\hline & 3 & 600 & & & & 100 & & 20 & ROCK DOVE \\
\hline 1 & & 3 & & & & 1 & $\overline{1}$ & & GREAT HORNED OWL \\
\hline & 0 & 3 & & 1 & & 1 & & 0 & SNOWY OWL \\
\hline 3 & & & 1 & & 1 & 1 & 11 & & GREAT GRAY OWL \\
\hline & & & & & & & & & SHORT-EARED OWL \\
\hline 4 & & 3 & 2 & 6 & 3 & 1 & 4 & 1 & DOWNY WOODPECKER \\
\hline 2 & & 2 & 3 & 9 & 6 & 6 & 6 & 1 & HAIRY WOODPECKER \\
\hline 2 & & & & & & & 1 & & THREE-TOED WOODPECKER \\
\hline 1 & & & & & & & & & BLACK-BACKED WOODPECKER \\
\hline & & & & & & & & & NORTHERN FLICKER (Y-s) \\
\hline 5 & & & & & & & 1 & & PILEATED WOODPECKER \\
\hline & & 125 & & & 1 & & & & HORNED LARK \\
\hline 15 & & & & & 5 & & 3 & & GRAY JAY \\
\hline 10 & & 5 & 2 & 8 & 11 & 2 & 18 & 1 & BLUE JAY \\
\hline 40 & 4 & 107 & 5 & 9 & 17 & 14 & 3 & 27 & BLACK-BILLED MAGPIE \\
\hline & 2 & & & & & & & & AMERICAN CROW \\
\hline 78 & 2 & & 6 & 24 & 22 & 16 & 56 & 12 & COMMON RAVEN \\
\hline 28 & 2 & 13 & 4 & 35 & 36 & 20 & 26 & 4 & BLACK-CAPPED CHICKADEE \\
\hline 31 & & & & & 1 & & 4 & & BOREAL CHICKADEE \\
\hline & & 19 & & & 1 & & 4 & & RED-BREASTED NUTHATCH \\
\hline & & & & 4 & 5 & & 8 & & WHITE-BREASTED NUTHATCH \\
\hline & & 1 & & & & & & & AMERICAN ROBIN \\
\hline & 100 & 327 & & & & 50 & 1 & 30 & BOHEMIAN WAXWING \\
\hline & & 1 & & & & & & & CEDAR WAXWING \\
\hline & & & & & & & & & NORTHERN SHRIKE \\
\hline & & 5 & & & & & & & EUROPEAN STARLING \\
\hline & & & & & & & & & HARRIS' SPARROW \\
\hline & & & & & 1 & & & & DARK-EYED JUNCO (S-C) \\
\hline 680 & 30 & 1037 & 22 & 50 & 400 & 30 & 0 & 0 & SNOW BUNTING \\
\hline 13 & & 2 & & 13 & 16 & 24 & 30 & & PINE GROSBEAK \\
\hline & & 114 & & & & & & & HOUSE FINCH \\
\hline 75 & & 22 & & & & 15 & 12 & & COMMON REDPOLL \\
\hline & & & & & 0 & & & & PINE SISKIN \\
\hline 5 & & & & 14 & 136 & 2 & 33 & & EVENING GROSBEAK \\
\hline 26 & 3 & 1561 & 12 & & 7 & 15 & & 0 & HOUSE SPARROW \\
\hline 1204 & 171 & 4057 & 57 & $\overline{174}$ & $\overline{671}$ & 319 & 242 & 96 & TOTAL INDIVIDUALS \\
\hline 26 & 12 & 27 & 8 & 12 & 18 & 21 & 22 & 8 & SPECIES ON COUNT DAY \\
\hline 26 & 14 & 29 & 8 & 13 & 19 & 21 & 23 & 11 & SPECIES COUNT PERIOD \\
\hline 29 & 0 & 9 & 0 & 0 & 0 & 12 & 1 & 0 & INDIVIDUALS IN TABLES $4 \& 5$ \\
\hline 4 & 0 & 5 & 0 & 0 & 0 & 3 & 1 & 0 & SPECIES IN TABLES $4 \& 5$ \\
\hline
\end{tabular}


Table 3-11. SPECIES RECORDED FROM NINE OR MORE LOCALITIES

$(0=a$ species recorded during count period but not seen on count day)

SPECIES

\section{MALLARD}

COMMON GOLDENEYE

BALD EAGLE

NORTHERN GOSHAWK

GOLDEN EAGLE

GRAY PARTRIDGE

RING-NECKED PHEASANT

SPRUCE GROUSE

RUFFED GROUSE

SHARP-TAILED GROUSE

ROCK DOVE

GREAT HORNED OWL

SNOWY OWL

GREAT GRAY OWL

SHORT-EARED OWL

DOWNY WOODPECKER

HAIRY WOODPECKER

THREE-TOED WOODPECKER

BLACK-BACKED WOODPECKER

NORTHERN FLICKER (Y-s)

PILEATED WOODPECKER

HORNED LARK

GRAY JAY

BLUE JAY

BLACK-BILLED MAGPIE

AMERICAN CROW

COMMON RAVEN

BLACK-CAPPED CHICKADEE

BOREAL CHICKADEE

RED-BREASTED NUTHATCH

WHITE-BREASTED NUTHATCH

AMERICAN ROBIN

BOHEMIAN WAXWING

CEDAR WAXWING

NORTHERN SHRIKE

EUROPEAN STARLING

HARRIS' SPARROW

DARK-EYED JUNCO (S-C)

SNOW BUNTING

PINE GROSBEAK

HOUSE FINCH

COMMON REDPOLL

PINE SISKIN

EVENING GROSBEAK

HOUSE SPARROW

TOTAL INDIVIDUALS

SPECIES ON COUNT DAY

SPECIES COUNT PERIOD

INDIVIDUALS IN TABLES $4 \& 5$

SPECIES IN TABLES $4 \& 5$

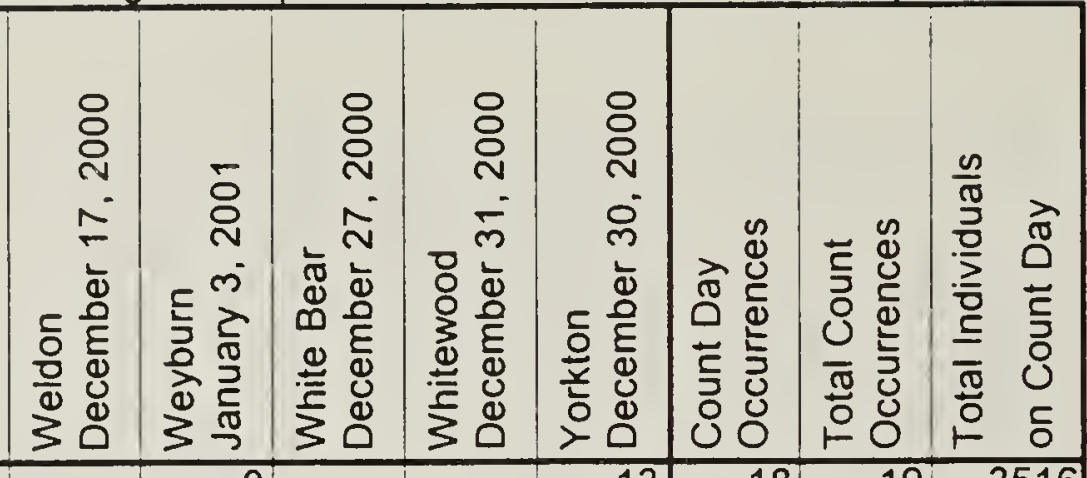

\begin{tabular}{|r|r|r|r|r|r|r|}
\hline & 0 & & 13 & 18 & 19 & 3516 \\
\hline & & & & 10 & 10 & 512 \\
\hline
\end{tabular}

\begin{tabular}{|c|c|c|c|c|c|c|c|}
\hline & & & & & 10 & 10 & 512 \\
\hline & & & & & 9 & 13 & 40 \\
\hline & & & & & 15 & 15 & 20 \\
\hline & & 2 & & & 14 & 15 & 19 \\
\hline & 54 & 28 & 39 & & 47 & 51 & 1627 \\
\hline & 0 & & & & 20 & 24 & 278 \\
\hline & & & & & 8 & 10 & 22 \\
\hline & & & & & 27 & 30 & \\
\hline
\end{tabular}

\begin{tabular}{|c|c|c|c|c|c|c|c|}
\hline 0 & & & $\overline{6}$ & 16 & 33 & 39 & 93 \\
\hline & 27 & 42 & 27 & & 54 & 57 & 1121 \\
\hline & 213 & 209 & 91 & 59 & 65 & 68 & 7033 \\
\hline
\end{tabular}

\begin{tabular}{|r|r|r|r|l|r|r|r|}
\hline & 5 & 1 & 2 & 39 & 45 & 89 \\
\hline & 8 & 0 & 1 & & 33 & 42 & 71 \\
\hline 0 & & & 1 & & 29 & 31 & 111 \\
\hline & & & & & 13 & 13 & 28 \\
\hline
\end{tabular}

\begin{tabular}{|r|r|r|r|r|r|r|r|}
\hline 2 & 2 & 0 & 32 & 7 & 73 & 78 & 431 \\
\hline 2 & 1 & & 16 & 8 & 76 & 78 & 363 \\
\hline
\end{tabular}

\begin{tabular}{|c|c|c|c|c|c|c|c|}
\hline & & & & & 8 & 8 & 10 \\
\hline & & & & & 8 & 9 & 9 \\
\hline & \multirow[t]{4}{*}{1} & & 2 & & 10 & 10 & 35 \\
\hline & & & 0 & & 16 & 24 & 29 \\
\hline & & 50 & 2 & & 24 & 24 & 1050 \\
\hline & & & & & 25 & 26 & 160 \\
\hline 5 & 0 & & 13 & 9 & 65 & 67 & 742 \\
\hline & 52 & $7 \epsilon$ & 147 & 87 & 95 & 95 & 4761 \\
\hline & & & & & $\mathrm{g}$ & $\overline{9}$ & 29 \\
\hline
\end{tabular}

\begin{tabular}{|r|r|r|r|r|r|r|r|}
\hline 3 & & & 34 & 56 & 75 & 77 & 2219 \\
\hline 6 & 5 & 9 & 335 & 53 & 95 & 97 & 4698 \\
\hline & & & & & 16 & 18 & 131 \\
\hline
\end{tabular}

\begin{tabular}{|c|c|c|c|c|c|c|c|}
\hline & & 5 & & 3 & 28 & 3 & 274 \\
\hline 2 & & 6 & & & 37 & 3 & 189 \\
\hline 1 & & 0 & & 2 & 9 & 1 & 25 \\
\hline
\end{tabular}

\begin{tabular}{|c|c|c|c|c|c|c|}
\hline & & & & & & \\
\hline & 0 & 0 & 719 & 44 & 52 & 12960 \\
\hline 0 & & 8 & 2 & 11 & 12 & 316 \\
\hline & & & & 13 & 15 & 16 \\
\hline 11 & 2 & 3 & & 28 & 30 & 415 \\
\hline
\end{tabular}

\begin{tabular}{|c|c|c|c|c|c|c|c|}
\hline & & & & & 8 & 8 & 12 \\
\hline & 6 & & & & 17 & 18 & 75 \\
\hline & 14 & 1 & 514 & 0 & 67 & 72 & 22834 \\
\hline \multirow[t]{2}{*}{12} & & & $\overline{0}$ & $\overline{18}$ & 60 & 61 & 1653 \\
\hline & & & & 0 & 11 & 16 & 501 \\
\hline \multirow[t]{2}{*}{4} & & & 1 & & 28 & 32 & 749 \\
\hline & & & & 0 & 13 & 18 & 267 \\
\hline \multirow[t]{2}{*}{4} & & & & & 30 & 32 & 2073 \\
\hline & 238 & 140 & 290 & 43 & 84 & 85 & 17659 \\
\hline 38 & 642 & 560 & 1575 & 1095 & & & 90334 \\
\hline 8 & 18 & 11 & 22 & 15 & & & \\
\hline 10 & 25 & 15 & 29 & 18 & & & \\
\hline 0 & 2 & 0 & 0 & 0 & & & \\
\hline 0 & 5 & 1 & 3 & 0 & & & \\
\hline
\end{tabular}


Figure 1. Location of 2000 counts (numbers correspond to locality names in Tables)

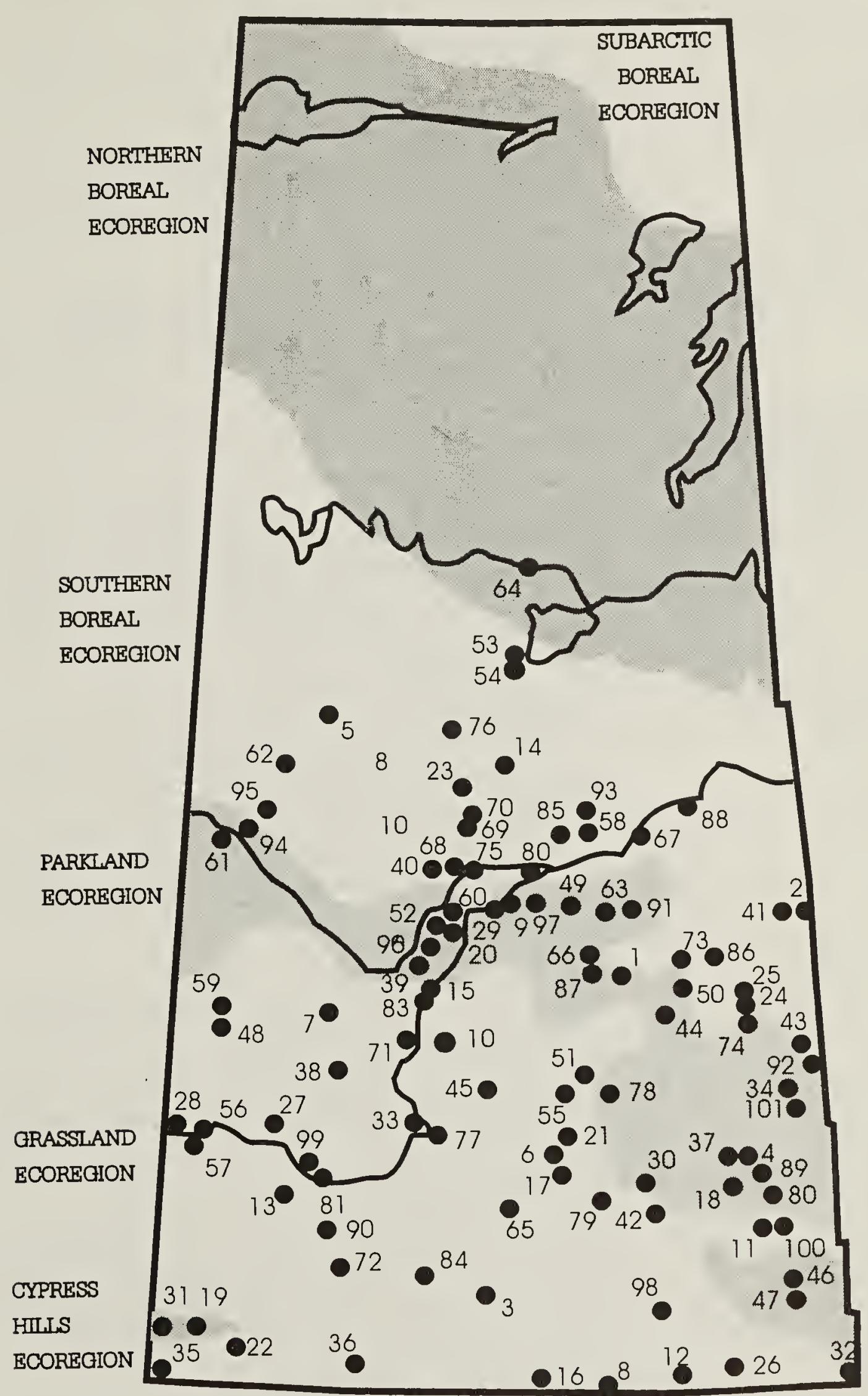


Table 4. Species recorded on eight or fewer counts

\begin{tabular}{|c|c|}
\hline Species & Locality and Number ( $0=$ count period species) \\
\hline CANADA GOOSE & $\begin{array}{l}\text { Biggar (0), Fort Qu'appelle (1), Gardiner Dam (52), } \\
\text { Regina (295), Round Lake (2), Saskatoon (1), Swift } \\
\text { Curent (0) }\end{array}$ \\
\hline NORTHERN PINTAIL & Coronach (1) \\
\hline GADWALL & Gardiner Dam (1) \\
\hline AMERICAN WIGEON & Estevan (1) \\
\hline CANVASBACK & Estevan (4) \\
\hline LESSER SCAUP & Coronach (1), Estevan (5) \\
\hline BUFFLEHEAD & Coronach (1) \\
\hline RED-BREASTED MERGANSER & Squaw Rapids (1) \\
\hline COMMON MERGANSER & $\begin{array}{l}\text { Coronach (1), Estevan (3), Fort Qu'appelle(0), } \\
\text { Gardiner Dam (250), Prince Albert (1), Round Lake } \\
\text { (2), Squaw Rapids (25) }\end{array}$ \\
\hline NORTHERN HARRIER & Estuary (1) \\
\hline SHARP-SHINNED HAWK & Pike Lake (1) \\
\hline COOPER'S HAWK & Saskatoon (2) \\
\hline ROUGH-LEGGED HAWK & $\begin{array}{l}\text { Bightwater Reservoir (1), Govenlock (2), Leader } \\
\text { South (1), Saskatoon (1), Weyburn (1) }\end{array}$ \\
\hline MERLIN & $\begin{array}{l}\text { Eastend (0), Harris (1), Moose Jaw (0), Regina (1), } \\
\text { Saskatoon (1), Swift Current (0), Weyburn (1), } \\
\text { Whitewood (0) }\end{array}$ \\
\hline PEREGRINE FALCON & Eastend (0) \\
\hline GYRFALCON & $\begin{array}{l}\text { Clark's Crossing (1), Gardiner Dam (3), Leader South } \\
\text { (1), Qu'appelle Valley Dam (1), Saskatchewan } \\
\text { Landing Provincial Park (1), Somme (0) }\end{array}$ \\
\hline PRAIRIE FALCON & $\begin{array}{l}\text { Big Muddy (1), Bromhead (1), Coronach (2), Estevan } \\
\text { (3), Eston (0), Leader South (1), White Bear (0) }\end{array}$ \\
\hline CHUKAR & Spalding $(0)$ \\
\hline SAGE GROUSE & Govenlock (4) \\
\hline WILD TURKEY & $\begin{array}{l}\text { Estuary (2), Fort Walsh (1), Gainsborough (23), } \\
\text { Kamsack (14), Kennedy (15) }\end{array}$ \\
\hline AMERICAN COOT & Coronach (3), Estevan (28) \\
\hline COMMON SNIPE & $\begin{array}{l}\text { Fort Walsh (1), Saskatchewan Landing Provincial } \\
\text { Park (1) }\end{array}$ \\
\hline EURASIAN COLLARED-DOVE & Moose Jaw (0), Regina (1) \\
\hline MOURNING DOVE & Harris (1), Luseland (0), Pike Lake (1) \\
\hline NORTHERN HAWK-OWL & $\begin{array}{l}\text { Armit (1), Maidstone Bridge (1), Meadow Lake (1), } \\
\text { Nisbet Forest W. (0), Saskatchewan River Forks (1), } \\
\text { Squaw Rapids (1), Turtleford (1), Turtle Lake (1) }\end{array}$ \\
\hline BARRED OWL & Snowden (1), Weyburn (0) \\
\hline BOREAL OWL & Emma Lake (0), Somme (0) \\
\hline NORTHERN SAW-WHET OWL & Good Spirit Lake (0), Preeceville (0), Whitewood (0) \\
\hline NORTHERN FLICKER (R-s) & Saskatchewan Landing Provincial Park (1) \\
\hline BROWN CREEPER & $\begin{array}{l}\text { Fort Walsh (1), Moose Jaw (0), Prince Albert (1), } \\
\text { Saskatoon (1) }\end{array}$ \\
\hline GOLDEN-CROWNED KINGLET & Craven (0), Fort Walsh (1), Regina (3), Saskatoon (1) \\
\hline TOWNSEND'S SOLITAIRE & Regina (1) \\
\hline
\end{tabular}


Table 4. Species recorded on eight or fewer counts

\begin{tabular}{|c|c|}
\hline Species & Locality and Number ( $0=$ count period species) \\
\hline VARIED THRUSH & Saskatoon (2), Weyburn (0) \\
\hline NORTHERN CARDINAL & Bangor $(0)$ \\
\hline SPOTTED TOWHEE & Saskatoon (1) \\
\hline AMERICAN TREE SPARROW & Fort Walsh (21), Kamsack (20), Ponteix (1) \\
\hline VESPER SPARROW & Whitewood (0) \\
\hline SONG SPARROW & Fort Walsh (2), Kinloch (1) \\
\hline WHITE-THROATED SPARROW & Saskatoon (5), Swift Current (2) \\
\hline DARK-EYED JUNCO (OREGON) & Regina (2) \\
\hline LAPLAND LONGSPUR & $\begin{array}{l}\text { Bromhead (3), Cabri (50), Govenlock (1), Shamrock } \\
(2)\end{array}$ \\
\hline RED-WINGED BLACKBIRD & Gardiner Dam (1), Govenlock (2), Weyburn (0) \\
\hline RUSTY BLACKBIRD & Estuary (2), Fort Walsh (5), Kennedy (1) \\
\hline BREWER'S BLACKBIRD & Govenlock (1) \\
\hline COMMON GRACKLE & $\begin{array}{l}\text { Hudson Bay (1), Leader North (1), Leader South (1), } \\
\text { Moose Jaw (0), Saskatoon (1), , Swift Current (5) }\end{array}$ \\
\hline BROWN-HEADED COWBIRD & Saskatoon (1), Turtleford (1) \\
\hline GRAY-CROWNED ROSY FINCH & Beauval (1), Fort Walsh (28) \\
\hline PURPLE FINCH & $\begin{array}{l}\text { Bangor (1), Fort Qu'appelle (0), Kenosee Lake (17), } \\
\text { Moose Jaw (2), Pike Lake (0), Swift Current (2) }\end{array}$ \\
\hline RED CROSSBILL & $\begin{array}{l}\text { Cypress Hills Provincial Park (6), Fort Walsh (4), Pike } \\
\text { Lake (2) }\end{array}$ \\
\hline WHITE-WINGED CROSSBILL & $\begin{array}{l}\text { Armit (4), Nipawin (4), Prince Albert (7), Saskatoon } \\
(7)\end{array}$ \\
\hline HOARY REDPOLL & $\begin{array}{l}\text { Love (5), Nisbet Forest W. (0), Preeceville (0), Squaw } \\
\text { Rapids (2), Turtleford (10) }\end{array}$ \\
\hline AMERICAN GOLDFINCH & Craven (0), Kenosee Lake (1), Saskatoon (1) \\
\hline
\end{tabular}

Table 5. Birds not identified to species

\begin{tabular}{|l|l|}
\hline Species & Locality and Number \\
\hline EAGLE species & Qu'Appelle Valley Dam (1) \\
\hline HAWK species & Prince Albert (1) \\
\hline WOODPECKER species & $\begin{array}{l}\text { Clark's Crossing (1), Hudson Bay (1), La Ronge } \\
\text { North (2) }\end{array}$ \\
\hline CROSSBILL species & Saskatoon (2) \\
\hline REDPOLL species & Craven (8) \\
\hline
\end{tabular}


Table 6. New and tying 2000 high counts (new highs in bold italics)

\begin{tabular}{|l|c|c|c|l|}
\hline LOCATION & $\begin{array}{c}2000 \\
\text { COUNT }\end{array}$ & SPECIES & $\begin{array}{c}\text { PREVIOUS } \\
\text { HIGH }\end{array}$ & LOCATION(YEAR) \\
\hline Estevan & 4 & CANVASBACK & 4 & Regina (1970) \\
\hline Pike Lake & 1 & SHARP-SINNED HAWK & 1 & 13 counts N. to Birch Hills \\
\hline Saskatoon & 2 & COOPER'S HAWK & 1 & 11 counts N. to Kamsack \\
\hline Gardiner Dam & 3 & GRYFALCON & 3 & Squaw Rapids (1988) \\
\hline Snowden & 18 & GREAT GRAY OWL & 8 & Squaw Rapids (1988) \\
\hline Saskatoon & 46 & DOWNY WOODPECKER & 37 & Saskatoon (1991) \\
\hline Saskatoon & 732 & BLACK-CAPPED CHICKADEE & 640 & Saskatoon (1998) \\
\hline Saskatoon & 2 & VARIED THRUSH & 1 & 4 counts N. to Saskatoon \\
\hline Saskatoon & 1 & SPOTTED TOWHEE & 1 & N. to Leader \\
\hline Fort Walsh & 2 & SONG SPARROW & 2 & Fort Qu'Appelle (1988) \\
\hline
\end{tabular}

Table 7. Population Status of selected species (based on birds per hour effort)

\begin{tabular}{|c|c|c|c|c|c|}
\hline Species & $\stackrel{\infty}{8} \stackrel{\infty}{\circ}$ & 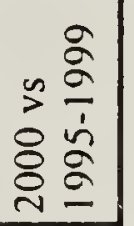 & Species & $\stackrel{2}{8} \stackrel{2}{\circ}$ & 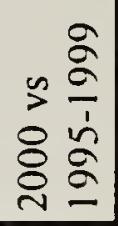 \\
\hline CANADA GOOSE & -98 & -98 & COMMON RAVEN & -6 & -3 \\
\hline MALLARD & -90 & -84 & BLACK-CAPPED CHICKADEE & 30 & 15 \\
\hline COMMON GOLDENEYE & -35 & -28 & BOREAL CHICKADEE & -13 & -16 \\
\hline BALD EAGLE & -57 & -54 & RED-BREASTED NUTHATCH & -45 & -51 \\
\hline NORTHERN GOSHAWK & 33 & 20 & WHITE-BREASTED NUTHATCH & 15 & -2 \\
\hline GOLDEN EAGLE & -54 & -54 & BROWN CREEPER & 0 & -61 \\
\hline MERLIN & -69 & -80 & GOLDEN-CROWNED KINGLET & -90 & -88 \\
\hline PRAIRIE FALCON & -20 & 44 & AMERICAN ROBIN & -63 & -76 \\
\hline GRAY PARTRIDGE & 101 & 2 & BOHEMIAN WAXWING & 220 & 32 \\
\hline RING-NECKED PHEASANT & 286 & 100 & CEDAR WAXWING & 25 & -7 \\
\hline SPRUCE GROUSE & -27 & 34 & NORTHERN SHRIKE & -16 & -24 \\
\hline RUFFED GROUSE & -24 & -32 & EUROPEAN STARLING & -28 & -22 \\
\hline SHARP-TAILED GROUSE & -2 & -16 & AMERICAN TREE SPARROW & -41 & 18 \\
\hline ROCK DOVE & 4 & -1 & DARK-EYED JUNCO & -69 & -70 \\
\hline GREAT HORNED OWL & 3 & -17 & LAPLAND LONGSPUR & -96 & -97 \\
\hline SNOWY OWL & 18 & -17 & SNOW BUNTING & 16 & 23 \\
\hline SHORT-EARED OWL & 64 & 158 & RUSTY BLACKBIRD & -43 & -71 \\
\hline DOWNY WOODDPECKER & 31 & 20 & PINE GROSBEAK & 123 & 5 \\
\hline HAIRY WOODPECKER & 12 & -3 & HOUSE FINCH & 17 & 68 \\
\hline THREE-TOED & 150 & -2 & RED CROSSBILL & -80 & -92 \\
\hline NORTHERN FLICKER & -10 & -16 & WHITE-WINGED CROSSBILL & -55 & -95 \\
\hline PILEATED WOODPECKER & -12 & 31 & COMMON REDPOLL & -96 & -91 \\
\hline HORNED LARK & -36 & -61 & HOARY REDPOLL & -94 & -87 \\
\hline GRAY JAY & 9 & 7 & PINE SISKIN & 4 & 2 \\
\hline BLUE JAY & 61 & 5 & EVENING GROSBEAK & -13 & -2 \\
\hline BLACK-BILLED MAGPIE & -7 & -12 & HOUSE SPARROW & -9 & -19 \\
\hline
\end{tabular}

